Document downloaded from:

http://hdl.handle.net/10251/136496

This paper must be cited as:

Ballesteros-Perez, P.; González-Cruz, M.; Cañavate-Grimal, A. (2013). On Competitive Bidding: Scoring and Position Probability Graphs. International Journal of Project Management. 31(3):434-448. https://doi.org/10.1016/j.jproman.2012.09.012

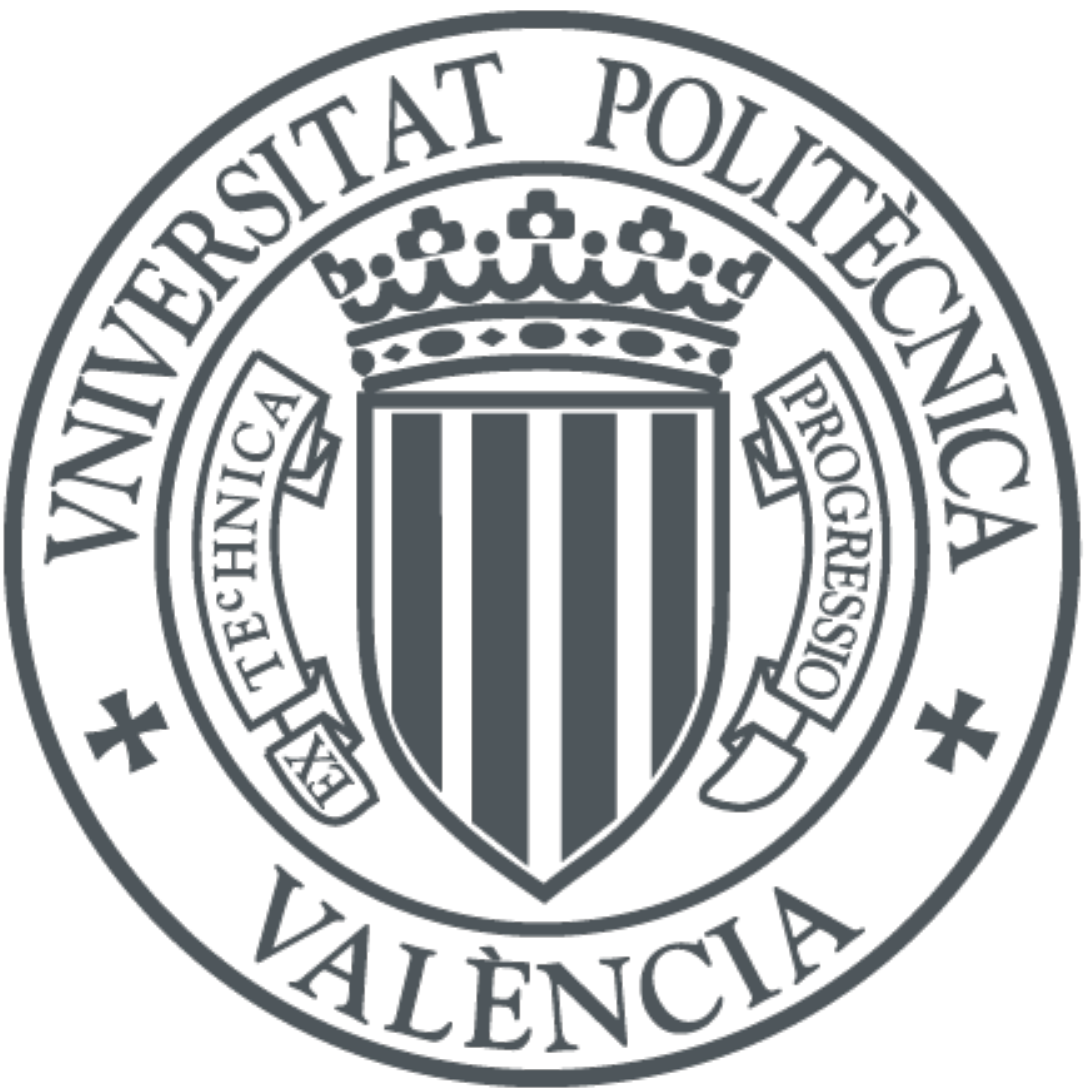

The final publication is available at

http://dx.doi.org/10.1016/j.ijproman.2012.09.012

Copyright Elsevier

Additional Information 


\section{ON COMPETITIVE BIDDING: SCORING AND POSITION PROBABILITY GRAPHS}

\section{Additional Information:}

Bidding (Contractors); Managing Proposal

Industry and Sector Specific: Engineering and Construction.

\section{Authors:}

Ballesteros-Pérez, Pablo ${ }^{\text {a }}$; González-Cruz, Mª Carmen $^{\text {b }}$; Cañavate-Grimal, Antonio ${ }^{\text {c }}$

a Dpto. de Ingeniería y Gestión de la Construcción

Facultad de Ingeniería. Universidad de Talca

Camino los Niches, km 1. Curicó (Chile)

Email: pballesteros@utalca.cl ; pabbalpe@hotmail.com

Phone: (+56) 75201733 Fax: (+56) 75325958

b Lecturer in Departamento de Proyectos de Ingeniería. Escuela Técnica Superior de Ingenieros Industriales. Universitat Politècnica de València. $C^{\circ}$ de Vera s/n, 46022 Valencia, Spain

$\mathrm{PhD}$. Industrial Engineering. IPMA certified Project Manager (C level)

Phone: +34 963879866 (ext.:75654 ) Fax: +34 963879869 (ext.:79869 ).

E-mail: $\underline{\text { mcgonzal@dpi.upv.es }}$

Corresponding author

c Lecturer in Departamento de Ingeniería de la Edificación y Producción Industrial. Escuela Superior de Enseñanzas Técnicas (ESET). Universidad CEU Cardenal Herrera. Calle de San Bartolomé 55. 46115. Alfara del Patriarca, Valencia. Spain.

MSc. Civil Engineering \& Geotechnical Engineering

Phone: +34961369000 Fax: +34961300977

E-mail: antonio.canavate@uch.ceu.es 


\title{
On Competitive Bidding: Scoring and Position Probability Graphs
}

\begin{abstract}
Iso-Score Curves Graph (iSCG) and mathematical relationships between Scoring Parameters (SP) and Forecasting Parameters (FP) can be used in Economic Scoring Formulas (ESF) used in tendering to distribute the score among bidders in the economic part of a proposal.
\end{abstract}

Each contracting authority must set an ESF when publishing tender specifications and the strategy of each bidder will differ depending on the ESF selected and the weight of the overall proposal scoring.

The various mathematical relationships and density distributions that describe the main SPs and FPs, and the representation of tendering data by means of iSCGs, enable the generation of two new types of graphs that can be very useful for bidders who want to be more competitive: the Scoring and Position Probability Graphs.

Keywords: bid; tender; auction; construction; score, forecast.

\section{Introduction}

Competitive bidding is a transparent procurement method in which bids from competing contractors, suppliers, or vendors are invited by openly advertising the scope, specifications, and terms and conditions of the proposed contract; as well as the criteria by which the bids will be evaluated. Competitive bidding aims at obtaining goods and services at the lowest price by stimulating competition and preventing favoritism.

Research in the area of competitive bidding strategy models has been in progress since the 1950s (Deltas \& Engelbrecht-Wiggans, 2005; Dikmen et al., 2007; Engelbrecht-Wiggans, 1980, 1989; Harstad \& Saša Pekec, 2008; Lo et al. 2007; Naoum, 1994; Näykki, 1976; Rothkopf \& Harstad, 1994; Rothkopf, 1969; Ye et al., 2008).

Competitive bidding strategy models have been developed to predict the probability of a bidder winning an auction (Engelbrecht-Wiggans, 1980; Näykki, 1976) or being awarded a project (Ravanshadnia et al., 2010; Vergara, 1977). Most of these models are based on the theory of Games, Decision Analysis and Operational Research and are difficult to apply to real-world business contexts given the complex mathematical formulations used in the models (Engelbrecht-Wiggans, 1980; Harstad \& Saša Pekec, 2008; Rothkopf \& Harstad, 1994). 
Because of the multiple technical and financial criteria involved in public tendering (Engelbrecht-Wiggans, 1980; Fayek, 1998; Harstad \& Saša Pekec, 2008; Näykki, 1976; Rothkopf \& Harstad, 1994; Skitmore \& Drew, 2001; Skitmore, 2002, 2004) there is still a need for the development of new tools to help decision makers and improve the selection process for bidders (Watt et al., 2009).

Ballesteros-Pérez, 2010 devised, and Ballesteros-Pérez et al., 2012a subsequently extended, the iso-Score Curve Graph (iSCG) as a practical tool to help bidders improve their competitive bidding strategies and increase their chances of winning a contract. This tool enables bidders to place their bids using simple statistical procedures based on previous bidding experiences sharing the same Economic Scoring Formula (ESF).

The economic criterion is usually one of the most important evaluation criteria and Economic Scoring Formulae are used to rate different proposals. The variables of these formulae are termed Scoring Parameters (SP) (Ballesteros-Pérez et al., 2012b). To predict SPs, some other parameters, which are termed Forecasting Parameters (FP) (Ballesteros-Pérez et al., 2012b), must be measured. This paper takes advantage of the new Bid Tender Forecasting Model (BTFM) which is based on the relationship between Scoring and Forecasting Parameters (SPs and FPs respectively) previously studied in Ballesteros-Pérez et al., 2012a and 2012b, and, furthermore, it is also shown how the different variables and results can be plotted on two new types of graphs: the Scoring Probability Graph (SPG) and the Position Probability Graph (PPG).

The BTFM equations described and implemented in this paper have been derived from capped tenders so far. Capped tender contract value is upper-limited by the contracting authority and this limitation is clearly stated in the tender specifications. Hence, all bidders must underbid that estimation. Nevertheless, the graphs presented later will not be restricted to these tenders once new equations are found to be valid in uncapped tendering contexts.

\section{Background}

Despite the extensive literature on the theory of auctions and competitive bidding for contract tendering, most models are based on theoretical assumptions that are difficult to apply to real cases (Skitmore, 2008). Bidding theory and strategy models (see Stark and Rothkopf, 1979, for an early bibliography) frequently make use of the so-called the statistical hypotheses' because auction bids are assumed to contain statistical properties such as fixed parameters and randomness (Skitmore, 2002).

The first studies (e.g., Friedman, 1956) assumed that each bidder drew bids from a probability distribution unique to that bidder, with low-frequency bidders being pooled as a special case. Pim (1974) analyzed a number of projects awarded to four American 
construction companies. His study indicated that the average number of projects awarded is proportional to the reciprocal of the average number of bidders competing - the proportion that would be expected to be won by pure 'chance' alone. That suggested an extremely simple 'equal probability' model in which the expected probability of entering the lowest bid in a k-size auction, that is, an auction in which $\mathrm{k}$ bidders enter bids, is the reciprocal of $\mathrm{k}$.

McCaffer and Pettitt (1976) and Mitchell (1977) assumed non-unique and homogeneous probability distributions, enabling a suitable distribution shape to be empirically fitted (uniform, in the case of McCaffer and Pettitt) and the derivation of other statistics based on an assumed (Normal) density function

Since then, different models have been developed to calculate the probability, $\operatorname{Pr}(m)$, of individual bidders winning an auction (Skitmore et al., 2007) but only in uncapped tendering: Friedman, 1956 assumed either interdependence or perfect estimation; Gates, 1967 used a Weibul probability distribution function; Carr, 1982, assumed homogeneous variances; and Skitmore, 1991, used a lognormal probability distribution function; among others.

A major problem is that in the context of construction contract bidding, it is difficult to collect the necessary data of each bidder for predictions to be effective (Skitmore, 2002). Moreover, Skitmore showed that the homogeneity assumption (Skitmore, 1991) was untenable for real datasets of construction contract auctions, at least insofar as its superiority in predicting the probability of lowest bidders is concerned (Skitmore, 1999), and Runeson and Skitmore (1999) cast doubt on the whole future of the heterogeneous approach to modeling construction contract auction bids on the basis of its necessary, but forced, assumption of temporal invariance (fixed parameters) in the absence of the lengthy repeated trials assumed by the statistical model - each bidder not bidding frequently enough to generate a reasonable size dataset.

The Bid Tender Forecasting Graphs proposed in this paper are based on previous research developed by Ballesteros-Pérez et al., 2012a and 2012b and solve, although only in capped tendering for the time being, the main problems encountered in previous models (Skitmore \& Runeson, 2006) as it enables (1) studying bidding behaviors with a significantly smaller database than previous works; (2) forecasting the probability of obtaining a particular score and/or position among competitors; (3) analyzing time variations between tenders, and (4) measuring the tender forecast performance.

Items (1) and (3) allow the present BTFM to use a heterogeneous approach based on parameters that vary over time (not on fixed parameters as suggested by Skitmore, 1999, and Runeson and Skitmore, 1999). 
Finally, although other formal and analytical risk models have recently been developed to prescribe how risk is to be incorporated into construction bids (Egemen \& Mohamed, 2007; Han et al., 2005; Hartono \& Yap, 2011; Mohamed et al., 2011; Oo et al., 2008a, 2008b), in practice, price risks are usually excluded from the final bid (or at least subjectively biased) in order to improve competitiveness (Banki et al, 2008; Han et al., 2005; Laryea \& Hughes, 2011). The BTFM that will be developed by the authors does not consider risk issues yet, and so, risk models may be a useful complement to the proposed forecasting model.

\section{Basic definitions}

Spanish tendering terminology is mainly used as this study was carried out in Spain, although some new terms are included. The BTFM described in this paper has been applied to capped tenders, that is, tenders upper-limited to the contracting authority estimate stated in the tender specifications.

However, the graphs shown below will not be only restricted to this type of tenders. If the main mathematical relationships between SPs and FPs are re-written to leave out the tender amount/price (A), the graphs shown below could also be used for both capped and noncapped tenders, since this latter type of tender is the most common in many countries

For the sake of clarity, we will define some terms used in this paper (see Appendix A for further information).

'Economic Scoring Formula' (ESF) refers to the mathematical expressions used to assign numerical scores to each bidder from the bid price expressed on a monetary-unit basis. ESF comprises the mathematical operations that provide the score and the mathematical expression that determines which bids are abnormal or risky (Abnormally Low Bids Criteria (ALBC). ALBC has received much less attention in the literature than analysis of bidding behaviors (Chao \& Liou, 2007).

'Bidder's Drop' $\left(D_{i}\right)$. It is the discount or bid reduction on the initial price of a contract $(A)$ submitted by a given contractor $i$ for a particular capped tender. It is mathematically expressed as:

$$
D_{i}=1-\frac{B_{i}}{A}
$$

Where $D_{i}$ is the Drop (expressed in per-unit values) of bidder ' $i$ ', $B_{i}$ is the Bid (expressed in monetary values) of bidder ' $\mathrm{i}$ ', and $\mathrm{A}$ is the initial Amount of money (in monetary value) of the tender (generally set by the contracting authority in many countries).

In Spanish tendering practice, when referring to bid amounts, it is usual to use a discount on the contract value A. This discount is called in Spanish 'baja', meaning literally fall or drop. 
This term has been translated merely as 'Drop' because no similar concept has been found in the international bibliography.

The ESF scores are obtained by either using the bidders' bids $\left(B_{i}\right)$ in monetary values or converting bids into drops $\left(D_{i}\right)$ in per-unit values. However, for the comparison of bids in different bidding processes with different initial bid amounts $(A)$ for each tender, it is preferable to use Drops $\left(D_{i}\right)$ rather than monetary-based Bids $\left(B_{i}\right)$.

'Scoring Parameter' (SP). SPs are the variables used in ESFs and are calculated from the distribution of the bids participating in a tender contest. The main SPs are: mean Drop $\left(D_{m}\right)$, maximum Drop $\left(D_{\max }\right)$; minimum Drop $\left(D_{\min }\right)$; Drops' standard deviation $(\sigma)$ and abnormal Drop ( $D_{a b n}$ ) (see Ballesteros-Pérez et al., 2012b or Appendix B 'Relationship between SP monetary-based and Drop-based values' for further information)

'Forecasting Parameters' (FP). FP refers to the variables that can be estimated in advance before the tender submission deadline. Their values must be closely related to the SPs to be useful. In this study the variable 'Estimated Cost' $\left(D_{0}\right)$ is used as an FP calculated in per-unit Drop rather than monetary value.

Some recent conceptual models have also been developed for use by contractors as part of a more reliable approach to identifying key competitors and as a basis for formulating bidding strategies (Oo et al., 2008a, 2010). Competitiveness between bids is examined using linear mixed models that employ variables such as project type and size; work sector; work nature; market conditions; as well as number of bidders (Drew \& Skitmore, 1997; Oo, et al., 2008a, 2008b). Some of these variables can also be considered as FPs (project type and size; work sector; work nature; market conditions) but are difficult to quantify.

\section{Tendering specifications review}

Ballesteros-Pérez, 2010 (Annex I) reviewed 120 tender specifications documents from Spanish Public Administrations and private companies in order to study the scoring parameters (SPs) and Economic Scoring Formulas (ESFs) used in Spain.

The review is quite representative of the Spanish tendering system, as it comprises: tenders and auctions from private companies and public administrations (city councils, local councils, semi-public entities, universities, ministries, etc), it also includes a wide variety of civil engineering works and services from various geographical regions (including the islands) and features a wide range of economic tender amounts. Although the sample only contains Spanish tender documents, the variables analyzed are directly applicable to any country where requesting administrations or contracting authorities set an initial tender amount $(A)$ against which candidates must underbid (capped tendering or upper-limited-price tendering). 
Among the wide range of tender documents collected, several contracting authorities generated sufficient tendering processes to enable an in-depth statistical analysis. Although the results obtained from these contracting authorities were very similar, a sub-dataset from one public administration was selected in order to illustrate through a numeric example how the mathematical relationships between SPs and one FP (Estimated Cost or $D_{0}$, in this case) allows any bidding practitioner to draw up two new kinds of bid tender forecasting graphs.

The selected public administration is the 'Agencia Catalana del Agua' (Catalan Water Agency), ACA hereinafter, a semi-public administration that manages most of the water supply system in the Catalan region of Spain. ACA managed 51 construction tenders in approximately one year (from May 2007 to June 2008) and used the same ESF in its tender specifications (see Ballesteros-Pérez, 2010, Annex II).

\section{Drawing the Scoring Probability Graph (SPG)}

To create the SPG, the following 5-step procedure is proposed (Ballesteros-Pérez, 2010; Ballesteros-Pérez et al., 2012a):

1. Obtain and screen previous tenders that are as similar as possible to the tender to be forecasted (sharing the same ESF is a must).

2. Calculate SPs and FPs regression coefficients.

3. Specify the Estimated Cost $\left(D_{0}\right)$ in SP regression equations.

4. Draw the iso-Score Curve Graph (iSCG)

5. Draw the Scoring Probability Graphs (SPG)

These five steps will be explained in detail when developing a numeric example based on a real ACA tendering dataset.

\section{$\underline{5.1 . \text { Analysis of previous tenders }}$}

An historical dataset is necessary for any forecast, otherwise it is difficult to calculate a proper prediction. Every tender must include a register of, at least, the following data: tender code / ID; tender deadline; nature of work, economic tender amount, number of bidders $(\mathrm{N})$, mean Drop $\left(D_{m}\right)$, maximum Drop $\left(D_{\max }\right)$, minimum Drop $\left(D_{\min }\right)$, bidders' Bids' standard deviation $(\sigma)$ and estimated Cost $\left(D_{0}\right)$. 
Furthermore, concerning BTFM it is necessary to start with a collection of previous tenders that are homogeneous with the tender to be forecasted. By the term 'homogeneous' we understand that previous and future tenders must be identical or very similar in terms of: scope of works, ESF and geographical region.

Moreover, it is also desirable that the tender datasets are fairly recent, otherwise it will be necessary to check if there has been a time variation, because of changes in the economic situation or the potential competition. Finally, sharing the same contracting authority is advantageous.

The bidder who plans to forecast must have taken part in a minimum of three analyzed tenders so that the connection between FPs and SPs, can be calculated and some previous tenders estimated costs known ( $D_{0}$ values).

In this example, a bidder intends to bid for the construction of a Waste Water Treatment Plant (WWTP) with a tender amount around $€ 4.5$ million. The tender has been published by public Spanish Administration 'ACA' and the deadline is June 2008. With this data we can use a real historical and homogeneous 9-tender sub-dataset as presented in Table 1.

\begin{tabular}{|c|c|c|c|c|c|c|c|c|c|c|c|c|c|c|}
\hline Tender Code & ID & Tender Deadline & Nature of Work & Tender Amount ( $€$ ) & Dmax & $\mathrm{Dm}$ & Dmin & $\sigma$ & Do & $a(D \max )^{\top}$ & $\mathrm{b}(\mathrm{Dmin})^{\top}$ & $c(\sigma)$ & $d(D o)^{\top}$ & N \\
\hline Ст08000389 & 8 & 2008-03-31 & WWTP & $4,745,844.66 €$ & 0.2732 & 0.1813 & 0.0514 & 0.0491 & 0.1170 & -0.6193 & 0.8751 & 0.1277 & 1.0786 & 22 \\
\hline Ст07002822 & 18 & $2008-01-23$ & WWTP & $2,279,367.16 €$ & 0.2367 & 0.1854 & 0.1028 & 0.0381 & 0.0700 & -0.3398 & 0.5470 & 0.0991 & 1.1416 & 14 \\
\hline СТ07002921 & 19 & $2008-01-23$ & WWTP & $4,346,995.62 €$ & 0.2833 & 0.2262 & 0.1818 & 0.0383 & & -0.3268 & 0.2533 & 0.1001 & & 14 \\
\hline СТ07002108 & 24 & 2007-12-11 & WWTP & $5,208,624.36 €$ & 0.2390 & 0.1765 & 0.0955 & 0.0386 & 0.1210 & -0.4301 & 0.5573 & 0.1003 & 1.0674 & 22 \\
\hline СТ07001934 & 27 & 2007-09-17 & WWTP & $6,557,087.95 €$ & 0.2200 & 0.1277 & 0.0325 & 0.0530 & 0.1120 & -0.8282 & 0.8547 & 0.1409 & 1.0181 & 16 \\
\hline СТ07001972 & 28 & $2007-09-17$ & WWTP & $8,764,690.65 €$ & 0.2800 & 0.2097 & 0.1550 & 0.0428 & & -0.4246 & 0.3299 & 0.1112 & & 10 \\
\hline Ст07002052 & 29 & 2007-09-17 & WWTP & $6,217,700.13 €$ & 0.2985 & 0.2361 & 0.1489 & 0.0561 & & -0.3459 & 0.4837 & 0.1468 & & 9 \\
\hline СТ07001903 & 33 & $2007-08-30$ & WWTP & $2,773,494.15 €$ & 0.1500 & 0.0855 & 0.0105 & 0.0523 & 0.0350 & -0.8248 & 0.9592 & 0.1474 & 1.0552 & 9 \\
\hline СТ07001602 & 36 & $2007-08-20$ & WWTP & $3,489,863.47 €$ & 0.2450 & 0.1199 & 0.0374 & 0.0666 & & -1.1863 & 0.7816 & 0.1785 & & 9 \\
\hline \multirow{4}{*}{ 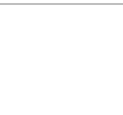 } & & & & & & & & \multirow{3}{*}{\multicolumn{2}{|c|}{ Average }} & $a m$ & bm & $\mathrm{Cm}$ & $d m$ & $\mathrm{Nm}$ \\
\hline & & & & & & & & & & -0.59 & 0.63 & 0.13 & 1.07 & 13.89 \\
\hline & & & & & & & & & & ao & bo & Co & do & $\mathrm{No}$ \\
\hline & & & & & & & & & Desvest: & 0.30 & 0.25 & 0.03 & 0.05 & 5.28 \\
\hline
\end{tabular}

Table 1: ACA historical tender sub-dataset

It can be observed that the deadlines, nature of work, and tender amounts are very similar to the tender to be forecasted.

The ESF and the ALBC used by the Administration for these tenders were always the same and coincident with those of the future tender (see Appendix $A$ to find the meaning of each variable):

$$
\mathrm{ESF}: S_{i}=1-\frac{B_{i}-B_{\min }^{*}}{B_{\max }-B_{\min }^{*}} \quad \text { ALBC: } B_{a b n}=B_{m}-2 S
$$




\subsection{Calculation of SP and FP regression coefficients}

Once the previous tender dataset is available and has been filtered to select past tenders as similar as possible to the tender to be forecasted, the next step is to know which particular regression coefficient values have the mathematical relationships that interconnect SPs and FPs (see 'Regression equations between SPs and FPs' in Appendix B).

These coefficients are named: $a, b, c$ and $d$, and their partial and final results are shown in the last five columns in Table 1 (the mathematical expressions applied are shown in Appendix B, as 'Expressions for calculating regression equation coefficient values). In Ballesteros-Pérez, 2012b it was demonstrated that these coefficient variations follow a Normal distribution, so their dispersion can be studied by means of their respective averages and standard deviations.

\subsection{Future tender's Estimated Cost $\left(\mathrm{D}_{0}\right)$ specification}

Regarding this variable, the only factor we consider to be significant is that the calculation must always be made in the same way, which means: being calculated by the same person or the same group of people with the same criteria; and always aggregating the same type of costs (taxes, indirect costs, structure cost from the company, etc). If a mark-up were included in $D_{0}$, then, when $D_{0}$ is calculated again it should include the same mark-up percentage.

Finally, the type of works and the ESF must be the same in every case. When these items suffer an important change, the historical $D_{0}$ data will be deleted and it is necessary to start from scratch correlating $\mathrm{D}_{0}$ with the SPs for future tenders, i.e., determining $a, b, c$ and $d$ values again.

In other words, accuracy and homogeneity while estimating costs are both important, even though this cost differs for each company. Observing these few homogeneity rules, $D_{0}$ values can be studied by means of a Normal distribution (Ballesteros-Pérez, 2012b).

Once the future tender $D_{0}$ is calculated by the bidder who is going to forecast (in our example, the estimated cost equals $D_{0}=0.25$ ), its value must be introduced in the BTFM regression expressions (see 'BTFM regression expressions as a function of $D_{0}$ ' and 'Expressions for calculating asterisk regression coefficient values' in Appendix B). Table 2 and Figure 1 show the main results, where ' $n_{\sigma}$ ' represents the multiples of standard deviation that are related to a particular accumulated Standard Normal Distribution probability (axis $Y$ ) and ' $h$ ' represents a coefficient that takes into account the number of dimensions involved in the multivariate Normal Distribution (see Appendix A) 


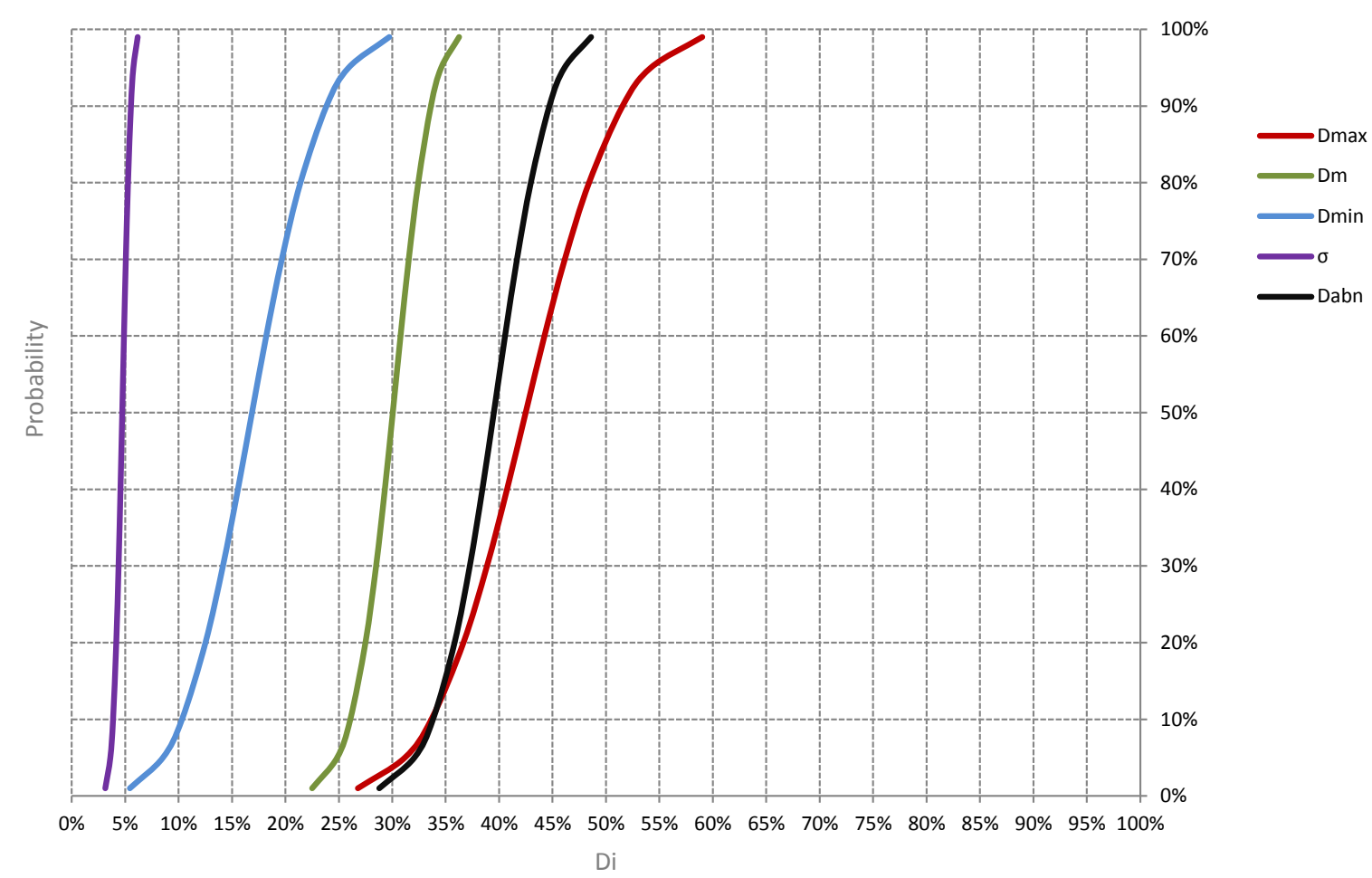

Figure 1: $D_{m}, D_{\max }, D_{\min }, \sigma$ and $D_{\text {abn }}$ probability curves for $D_{0}=0.25$

In Table 2, Scoring Parameter $D_{a b n}$ has also been calculated. Values depend exclusively on $D_{m}$ and $\sigma$ for the ALBC shown as an example $\left(D_{a b n}=D_{m}+2 \sigma\right.$ once it has been transformed into drops). 


\begin{tabular}{|c|c|c|c|c|c|c|c|c|c|c|c|c|c|}
\hline Probability (axis Y) & 0.01 & 0.05 & 0.10 & 0.20 & 0.30 & 0.40 & 0.50 & 0.60 & 0.70 & 0.80 & 0.90 & 0.95 & 0.99 \\
\hline \multicolumn{14}{|c|}{ BTFM's Dm's regression curves } \\
\hline dm & 1.07 & 1.07 & 1.07 & 1.07 & 1.07 & 1.07 & 1.07 & 1.07 & 1.07 & 1.07 & 1.07 & 1.07 & 1.07 \\
\hline $\mathbf{h}$ (1 variable total) & 1.00 & 1.00 & 1.00 & 1.00 & 1.00 & 1.00 & 1.00 & 1.00 & 1.00 & 1.00 & 1.00 & 1.00 & 1.00 \\
\hline no & -2.32 & -1.65 & -1.28 & -0.84 & -0.52 & -0.25 & 0.00 & 0.25 & 0.52 & 0.84 & 1.28 & 1.65 & 2.32 \\
\hline do & 0.05 & 0.05 & 0.05 & 0.05 & 0.05 & 0.05 & 0.05 & 0.05 & 0.05 & 0.05 & 0.05 & 0.05 & 0.05 \\
\hline$d^{*}=d_{m}+h \cdot n_{\sigma} \cdot d_{\sigma}$ & 0.97 & 1.00 & 1.01 & 1.03 & 1.05 & 1.06 & 1.07 & 1.08 & 1.10 & 1.11 & 1.13 & 1.15 & 1.18 \\
\hline Dm (axis X) & 0.22 & 0.25 & 0.26 & 0.27 & 0.28 & 0.29 & 0.30 & 0.31 & 0.32 & 0.32 & 0.34 & 0.35 & 0.36 \\
\hline \multicolumn{14}{|c|}{ BTFM's Dmax's regression curves } \\
\hline am & -0.59 & -0.59 & -0.59 & -0.59 & -0.59 & -0.59 & -0.59 & -0.59 & -0.59 & -0.59 & -0.59 & -0.59 & -0.59 \\
\hline $\mathbf{h}$ (2 variables total) & 0.71 & 0.71 & 0.71 & 0.71 & 0.71 & 0.71 & 0.71 & 0.71 & 0.71 & 0.71 & 0.71 & 0.71 & 0.71 \\
\hline $\mathbf{n}_{\sigma}$ & 2.32 & 1.65 & 1.28 & 0.84 & 0.52 & 0.25 & 0.00 & -0.25 & -0.52 & -0.84 & -1.28 & -1.65 & -2.32 \\
\hline$a_{\sigma}$ & 0.30 & 0.30 & 0.30 & 0.30 & 0.30 & 0.30 & 0.30 & 0.30 & 0.30 & 0.30 & 0.30 & 0.30 & 0.30 \\
\hline$a^{*}=a m+h \cdot n_{\sigma} \cdot a \sigma$ & -0.10 & -0.24 & -0.32 & -0.41 & -0.48 & -0.54 & -0.59 & -0.64 & -0.70 & -0.77 & -0.86 & -0.94 & -1.08 \\
\hline $\mathrm{dm}$ & 1.07 & 1.07 & 1.07 & 1.07 & 1.07 & 1.07 & 1.07 & 1.07 & 1.07 & 1.07 & 1.07 & 1.07 & 1.07 \\
\hline h (2 variables total) & 0.71 & 0.71 & 0.71 & 0.71 & 0.71 & 0.71 & 0.71 & 0.71 & 0.71 & 0.71 & 0.71 & 0.71 & 0.71 \\
\hline $\mathrm{n}_{\sigma}$ & -2.32 & -1.65 & -1.28 & -0.84 & -0.52 & -0.25 & 0.00 & 0.25 & 0.52 & 0.84 & 1.28 & 1.65 & 2.32 \\
\hline$d_{\sigma}$ & 0.05 & 0.05 & 0.05 & 0.05 & 0.05 & 0.05 & 0.05 & 0.05 & 0.05 & 0.05 & 0.05 & 0.05 & 0.05 \\
\hline$d^{*}=d_{m}+h \cdot n_{\sigma} \cdot d_{\sigma}$ & 1.00 & 1.02 & 1.03 & 1.05 & 1.06 & 1.06 & 1.07 & 1.08 & 1.09 & 1.10 & 1.11 & 1.12 & 1.15 \\
\hline Dmax (axis $\mathrm{X})$ & 0.27 & 0.31 & 0.34 & 0.37 & 0.39 & 0.41 & 0.42 & 0.44 & 0.46 & 0.48 & 0.52 & 0.54 & 0.59 \\
\hline \multicolumn{14}{|c|}{ BTFM's Dmin's regression curves } \\
\hline$b_{m}$ & 0.63 & 0.63 & 0.63 & 0.63 & 0.63 & 0.63 & 0.63 & 0.63 & 0.63 & 0.63 & 0.63 & 0.63 & 0.63 \\
\hline h (2 variables total) & 0.71 & 0.71 & 0.71 & 0.71 & 0.71 & 0.71 & 0.71 & 0.71 & 0.71 & 0.71 & 0.71 & 0.71 & 0.71 \\
\hline $\mathrm{n}_{\sigma}$ & 2.32 & 1.65 & 1.28 & 0.84 & 0.52 & 0.25 & 0.00 & -0.25 & -0.52 & -0.84 & -1.28 & -1.65 & -2.32 \\
\hline bo & 0.25 & 0.25 & 0.25 & 0.25 & 0.25 & 0.25 & 0.25 & 0.25 & 0.25 & 0.25 & 0.25 & 0.25 & 0.25 \\
\hline $\mathbf{b}^{*}=\mathbf{b}_{m}+\mathbf{h} \cdot \mathbf{n}_{\sigma} \cdot \mathbf{b}_{\sigma}$ & 1.04 & 0.92 & 0.85 & 0.78 & 0.72 & 0.67 & 0.63 & 0.58 & 0.53 & 0.48 & 0.40 & 0.33 & 0.21 \\
\hline$d m$ & 1.07 & 1.07 & 1.07 & 1.07 & 1.07 & 1.07 & 1.07 & 1.07 & 1.07 & 1.07 & 1.07 & 1.07 & 1.07 \\
\hline h (2 variables total) & 0.71 & 0.71 & 0.71 & 0.71 & 0.71 & 0.71 & 0.71 & 0.71 & 0.71 & 0.71 & 0.71 & 0.71 & 0.71 \\
\hline $\mathrm{n}_{\sigma}$ & -2.32 & -1.65 & -1.28 & -0.84 & -0.52 & -0.25 & 0.00 & 0.25 & 0.52 & 0.84 & 1.28 & 1.65 & 2.32 \\
\hline do & 0.05 & 0.05 & 0.05 & 0.05 & 0.05 & 0.05 & 0.05 & 0.05 & 0.05 & 0.05 & 0.05 & 0.05 & 0.05 \\
\hline$d^{*}=d_{m}+h \cdot n_{\sigma} \cdot d_{\sigma}$ & 1.00 & 1.02 & 1.03 & 1.05 & 1.06 & 1.06 & 1.07 & 1.08 & 1.09 & 1.10 & 1.11 & 1.12 & 1.15 \\
\hline Dmin (axis X) & 0.05 & 0.09 & 0.10 & 0.13 & 0.14 & 0.16 & 0.17 & 0.18 & 0.20 & 0.21 & 0.24 & 0.26 & 0.30 \\
\hline \multicolumn{14}{|c|}{ BTFM's $\sigma$ 's regression curves } \\
\hline $\mathrm{Cm}$ & 0.13 & 0.13 & 0.13 & 0.13 & 0.13 & 0.13 & 0.13 & 0.13 & 0.13 & 0.13 & 0.13 & 0.13 & 0.13 \\
\hline h (2 variables total) & 0.71 & 0.71 & 0.71 & 0.71 & 0.71 & 0.71 & 0.71 & 0.71 & 0.71 & 0.71 & 0.71 & 0.71 & 0.71 \\
\hline $\mathrm{n}_{\sigma}$ & -2.32 & -1.65 & -1.28 & -0.84 & -0.52 & -0.25 & 0.00 & 0.25 & 0.52 & 0.84 & 1.28 & 1.65 & 2.32 \\
\hline C $\sigma$ & 0.03 & 0.03 & 0.03 & 0.03 & 0.03 & 0.03 & 0.03 & 0.03 & 0.03 & 0.03 & 0.03 & 0.03 & 0.03 \\
\hline$c^{*}=c_{m}+h \cdot n_{\sigma} \cdot c_{\sigma}$ & 0.08 & 0.10 & 0.10 & 0.11 & 0.12 & 0.12 & 0.13 & 0.13 & 0.14 & 0.14 & 0.15 & 0.16 & 0.17 \\
\hline $\mathrm{dm}$ & 1.07 & 1.07 & 1.07 & 1.07 & 1.07 & 1.07 & 1.07 & 1.07 & 1.07 & 1.07 & 1.07 & 1.07 & 1.07 \\
\hline $\mathbf{h}$ (2 variables total) & 0.71 & 0.71 & 0.71 & 0.71 & 0.71 & 0.71 & 0.71 & 0.71 & 0.71 & 0.71 & 0.71 & 0.71 & 0.71 \\
\hline $\mathrm{n}_{\sigma}$ & -2.32 & -1.65 & -1.28 & -0.84 & -0.52 & -0.25 & 0.00 & 0.25 & 0.52 & 0.84 & 1.28 & 1.65 & 2.32 \\
\hline$d_{\sigma}$ & 0.05 & 0.05 & 0.05 & 0.05 & 0.05 & 0.05 & 0.05 & 0.05 & 0.05 & 0.05 & 0.05 & 0.05 & 0.05 \\
\hline$d^{*}=d_{m}+h \cdot n_{\sigma} \cdot d_{\sigma}$ & 1.00 & 1.02 & 1.03 & 1.05 & 1.06 & 1.06 & 1.07 & 1.08 & 1.09 & 1.10 & 1.11 & 1.12 & 1.15 \\
\hline$\sigma(\operatorname{axis} X)$ & 0.03 & 0.04 & 0.04 & 0.04 & 0.04 & 0.05 & 0.05 & 0.05 & 0.05 & 0.05 & 0.06 & 0.06 & 0.06 \\
\hline \multicolumn{14}{|c|}{ BTFM's Dabn's curves } \\
\hline $\mathrm{Dm}$ & 0.22 & 0.25 & 0.26 & 0.27 & 0.28 & 0.29 & 0.30 & 0.31 & 0.32 & 0.32 & 0.34 & 0.35 & 0.36 \\
\hline$\sigma$ & 0.03 & 0.04 & 0.04 & 0.04 & 0.04 & 0.05 & 0.05 & 0.05 & 0.05 & 0.05 & 0.06 & 0.06 & 0.06 \\
\hline Dabn (axis X) & 0.29 & 0.32 & 0.34 & 0.36 & 0.37 & 0.38 & 0.40 & 0.41 & 0.42 & 0.43 & 0.45 & 0.46 & 0.49 \\
\hline
\end{tabular}

Table 2: $D_{m}, D_{\max }, D_{\min }, \sigma$ and $D_{a b n}$ probability curves calculations for $D_{0}=0.25$

\subsection{Draw the iso-Score Curves Graph (iSCG)}

Ballesteros-Pérez, 2010 and Ballesteros-Pérez et al., 2012a explain how an iSCG is built. Applied to our example, the procedure to generate iso-Score Curves from the ESF is as follows:

1. Express mathematically the Economic Scoring formula. In our example this would lead to: 


$$
S_{i}=1-\frac{B_{i}-B_{\min }^{*}}{B_{\max }-B_{\min }^{*}} \quad B_{a b n}=B_{m}-2 S
$$

2. Convert the ESF (when expressed in monetary units) into Drops (with parameters expressed in per-unit values):

$$
S_{i}=1-\frac{D_{\max }^{*}-D_{i}}{D_{\max }^{*}-D_{\min }} \quad D_{a b n}=D_{m}+2 \sigma
$$

3. Work out the ESF value of variable $D_{i}$ (Bidder's Drop):

$$
D_{i}=D_{\text {max }}^{*}-\left(1-S_{i}\right)\left(D_{\text {max }}^{*}-D_{\text {min }}\right)
$$

4. Represent the different iso-Score Curves graphically for the required and/or selected Score values $\left(\mathrm{S}_{\mathrm{i}}\right)$. In our example, Table 3 shows the Scores $\left(\mathrm{S}_{\mathrm{i}}\right)$ from 0 to 1 , placed equidistantly at 0.10 intervals (using the first three equations shown in Appendix $B$

\begin{tabular}{|c|c|c|c|c|c|c|c|c|c|c|c|c|c|c|c|c|}
\hline \multicolumn{5}{|c|}{ SPs } & \multirow{2}{*}{$\begin{array}{c}\text { D*max } \\
\min \\
\text { (Dmax;Dabn) }\end{array}$} & \multicolumn{11}{|c|}{$\mathrm{Si}$ (iso-Scoring Curves) } \\
\hline $\mathrm{Dm}$ & Dmax & Dmin & $\sigma$ & Dabn & & 1 & 0,9 & 0,8 & 0,7 & 0,6 & 0,5 & 0,4 & 0,3 & 0,2 & 0,1 & 0 \\
\hline 0,00 & 0,00 & 0,00 & 0,00 & 0,00 & 0,00 & 0,00 & 0,00 & 0,00 & 0,00 & 0,00 & 0,00 & 0,00 & 0,00 & 0,00 & 0,00 & 0,00 \\
\hline 0,01 & 0,02 & 0,00 & 0,03 & 0,06 & 0,02 & 0,02 & 0,01 & 0,01 & 0,01 & 0,01 & 0,01 & 0,01 & 0,01 & 0,01 & 0,01 & 0,00 \\
\hline 0,02 & 0,03 & 0,01 & 0,03 & 0,08 & 0,03 & 0,03 & 0,03 & 0,03 & 0,02 & 0,02 & 0,02 & 0,02 & 0,01 & 0,01 & 0,01 & 0,01 \\
\hline 0,03 & 0,05 & 0,01 & 0,04 & 0,10 & 0,05 & 0,05 & 0,04 & 0,04 & 0,04 & 0,03 & 0,03 & 0,03 & 0,02 & 0,02 & 0,02 & 0,01 \\
\hline 0,04 & 0,06 & 0,02 & 0,04 & 0,12 & 0,06 & 0,06 & 0,06 & 0,05 & 0,05 & 0,04 & 0,04 & 0,03 & 0,03 & 0,03 & 0,02 & 0,02 \\
\hline 0,05 & 0,08 & 0,02 & 0,04 & 0,13 & 0,08 & 0,08 & 0,07 & 0,07 & 0,06 & 0,05 & 0,05 & 0,04 & 0,04 & 0,03 & 0,03 & 0,02 \\
\hline 0,10 & 0,15 & 0,04 & 0,05 & 0,19 & 0,15 & 0,15 & 0,14 & 0,13 & 0,12 & 0,11 & 0,10 & 0,09 & 0,08 & 0,07 & 0,05 & 0,04 \\
\hline 0,15 & 0,23 & 0,07 & 0,05 & 0,25 & 0,23 & 0,23 & 0,21 & 0,19 & 0,18 & 0,16 & 0,15 & 0,13 & 0,12 & 0,10 & 0,09 & 0,07 \\
\hline 0,20 & 0,29 & 0,10 & 0,05 & 0,30 & 0,29 & 0,29 & 0,28 & 0,26 & 0,24 & 0,22 & 0,20 & 0,18 & 0,16 & 0,14 & 0,12 & 0,10 \\
\hline 0,25 & 0,36 & 0,13 & 0,05 & 0,35 & 0,35 & 0,35 & 0,33 & 0,30 & 0,28 & 0,26 & 0,24 & 0,22 & 0,20 & 0,18 & 0,15 & 0,13 \\
\hline 0,30 & 0,42 & 0,17 & 0,05 & 0,39 & 0,39 & 0,39 & 0,37 & 0,35 & 0,33 & 0,30 & 0,28 & 0,26 & 0,24 & 0,21 & 0,19 & 0,17 \\
\hline 0,35 & 0,48 & 0,21 & 0,05 & 0,44 & 0,44 & 0,44 & 0,42 & 0,39 & 0,37 & 0,35 & 0,32 & 0,30 & 0,28 & 0,25 & 0,23 & 0,21 \\
\hline 0,40 & 0,54 & 0,25 & 0,04 & 0,49 & 0,49 & 0,49 & 0,46 & 0,44 & 0,42 & 0,39 & 0,37 & 0,34 & 0,32 & 0,30 & 0,27 & 0,25 \\
\hline 0,45 & 0,60 & 0,29 & 0,04 & 0,53 & 0,53 & 0,53 & 0,51 & 0,48 & 0,46 & 0,44 & 0,41 & 0,39 & 0,37 & 0,34 & 0,32 & 0,29 \\
\hline 0,50 & 0,65 & 0,34 & 0,04 & 0,58 & 0,58 & 0,58 & 0,55 & 0,53 & 0,51 & 0,48 & 0,46 & 0,44 & 0,41 & 0,39 & 0,37 & 0,34 \\
\hline 0,55 & 0,70 & 0,39 & 0,03 & 0,62 & 0,62 & 0,62 & 0,60 & 0,57 & 0,55 & 0,53 & 0,51 & 0,48 & 0,46 & 0,44 & 0,42 & 0,39 \\
\hline 0,60 & 0,74 & 0,45 & 0,03 & 0,66 & 0,66 & 0,66 & 0,64 & 0,62 & 0,60 & 0,58 & 0,56 & 0,53 & 0,51 & 0,49 & 0,47 & 0,45 \\
\hline 0,65 & 0,78 & 0,51 & 0,03 & 0,71 & 0,71 & 0,71 & 0,69 & 0,67 & 0,65 & 0,63 & 0,61 & 0,59 & 0,57 & 0,55 & 0,53 & 0,51 \\
\hline 0,70 & 0,82 & 0,57 & 0,02 & 0,75 & 0,75 & 0,75 & 0,73 & 0,71 & 0,69 & 0,68 & 0,66 & 0,64 & 0,62 & 0,60 & 0,59 & 0,57 \\
\hline 0,75 & 0,86 & 0,63 & 0,02 & 0,79 & 0,79 & 0,79 & 0,77 & 0,76 & 0,74 & 0,73 & 0,71 & 0,70 & 0,68 & 0,66 & 0,65 & 0,63 \\
\hline 0,80 & 0,89 & 0,70 & 0,02 & 0,83 & 0,83 & 0,83 & 0,82 & 0,81 & 0,79 & 0,78 & 0,77 & 0,75 & 0,74 & 0,73 & 0,71 & 0,70 \\
\hline 0,85 & 0,93 & 0,77 & 0,01 & 0,87 & 0,87 & 0,87 & 0,86 & 0,85 & 0,84 & 0,83 & 0,82 & 0,81 & 0,80 & 0,79 & 0,78 & 0,77 \\
\hline 0,90 & 0,95 & 0,84 & 0,01 & 0,92 & 0,92 & 0,92 & 0,91 & 0,90 & 0,89 & 0,89 & 0,88 & 0,87 & 0,87 & 0,86 & 0,85 & 0,84 \\
\hline 0,95 & 0,98 & 0,92 & 0,00 & 0,96 & 0,96 & 0,96 & 0,95 & 0,95 & 0,95 & 0,94 & 0,94 & 0,94 & 0,93 & 0,93 & 0,92 & 0,92 \\
\hline 1,00 & 1,00 & 1,00 & 0,00 & 1,00 & 1,00 & 1,00 & 1,00 & 1,00 & 1,00 & 1,00 & 1,00 & 1,00 & 1,00 & 1,00 & 1,00 & 1,00 \\
\hline
\end{tabular}
'Regression equations between SPs and FPs' and the expression obtained in step $3)$.

Table 3: Calculations of the iso-Score Curve Graph for the ESF and ALBC examples

It must be pointed out that the ESF proposed by the requesting administration in our example gives a maximum score $(\mathrm{Si})$ to the bidder who proposes a maximum (but not abnormally high) drop. This is the reason why $\mathrm{D}_{\text {max }}^{*}$ is equal to the minimum value between $D_{\max }$ and $D_{a b n}$ for any probability level.

If Table 3 is represented as a function of variable $D_{m}$, the iSCG is drawn and the first of the BTFM graphs is obtained. 


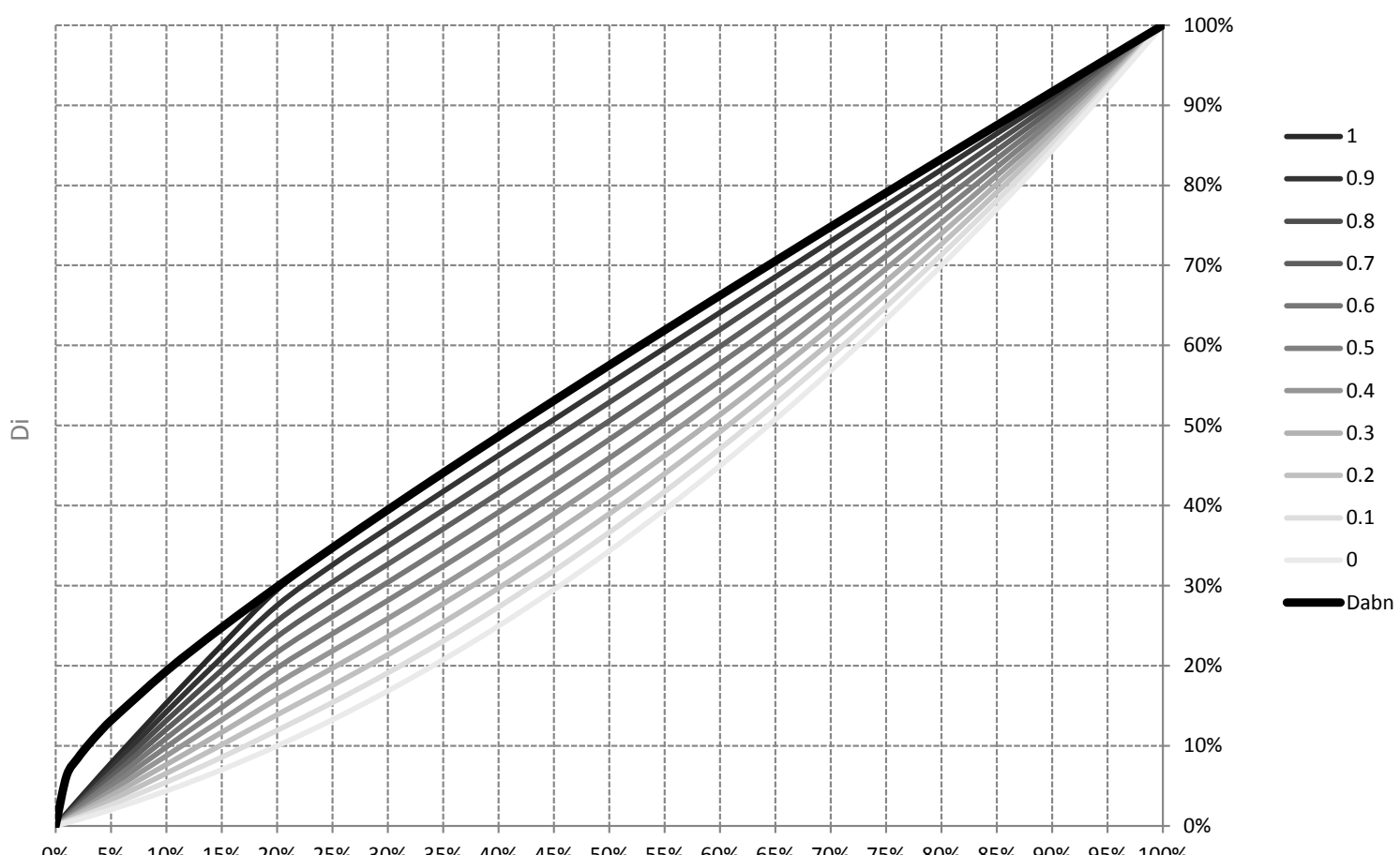

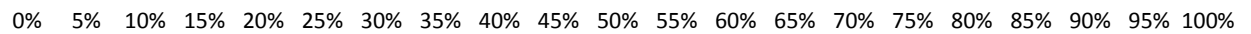

$\mathrm{Dm}$

Figure 2: iso-Score Curve Graph for the ESF and ALBC examples

\subsection{Drawing the Scoring Probability Graph (SPG)}

Once the ESF is calculated as a function of $D_{i}$ (see step 3 in previous sub-section), $D_{m}$, $D_{\max }, D_{\min }, \sigma$ and $D_{\text {abn }}$ Probability Curves values from Table 2 (figures in bold) or Figure 1 can be introduced in that expression, and the lower part of Table 4 is easily reached.

\begin{tabular}{|c|c|c|c|c|c|c|c|c|c|c|c|c|c|}
\hline$\downarrow S P \quad$ Prob $\rightarrow$ & 0.01 & 0.05 & 0.10 & 0.20 & 0.30 & 0.40 & 0.50 & 0.60 & 0.70 & 0.80 & 0.90 & 0.95 & 0.99 \\
\hline Dmax & 0.27 & 0.31 & 0.34 & 0.37 & 0.39 & 0.41 & 0.42 & 0.44 & 0.46 & 0.48 & 0.52 & 0.54 & 0.59 \\
\hline $\mathrm{Dm}$ & 0.22 & 0.25 & 0.26 & 0.27 & 0.28 & 0.29 & 0.30 & 0.31 & 0.32 & 0.32 & 0.34 & 0.35 & 0.36 \\
\hline Dmin & 0.05 & 0.09 & 0.10 & 0.13 & 0.14 & 0.16 & 0.17 & 0.18 & 0.20 & 0.21 & 0.24 & 0.26 & 0.30 \\
\hline$\sigma$ & 0.03 & 0.04 & 0.04 & 0.04 & 0.04 & 0.05 & 0.05 & 0.05 & 0.05 & 0.05 & 0.06 & 0.06 & 0.06 \\
\hline Dabn & 0.29 & 0.32 & 0.34 & 0.36 & 0.37 & 0.38 & 0.40 & 0.41 & 0.42 & 0.43 & 0.45 & 0.46 & 0.49 \\
\hline$D^{*} \max$ & 0.27 & 0.31 & 0.34 & 0.36 & 0.37 & 0.38 & 0.40 & 0.41 & 0.42 & 0.43 & 0.45 & 0.46 & 0.49 \\
\hline$\downarrow S i \quad$ Prob $\rightarrow$ & 0.01 & 0.05 & 0.10 & 0.20 & 0.30 & 0.40 & 0.50 & 0.60 & 0.70 & 0.80 & 0.90 & 0.95 & 0.99 \\
\hline 1.00 & 0.27 & 0.31 & 0.34 & 0.36 & 0.37 & 0.38 & 0.40 & 0.41 & 0.42 & 0.43 & 0.45 & 0.46 & 0.49 \\
\hline 0.90 & 0.25 & 0.29 & 0.31 & 0.33 & 0.35 & 0.36 & 0.37 & 0.38 & 0.39 & 0.41 & 0.43 & 0.44 & 0.47 \\
\hline 0.80 & 0.23 & 0.27 & 0.29 & 0.31 & 0.33 & 0.34 & 0.35 & 0.36 & 0.37 & 0.39 & 0.41 & 0.42 & 0.45 \\
\hline 0.70 & 0.20 & 0.24 & 0.27 & 0.29 & 0.30 & 0.32 & 0.33 & 0.34 & 0.35 & 0.36 & 0.38 & 0.40 & 0.43 \\
\hline 0.60 & 0.18 & 0.22 & 0.24 & 0.27 & 0.28 & 0.29 & 0.30 & 0.32 & 0.33 & 0.34 & 0.36 & 0.38 & 0.41 \\
\hline 0.50 & 0.16 & 0.20 & 0.22 & 0.24 & 0.26 & 0.27 & 0.28 & 0.29 & 0.31 & 0.32 & 0.34 & 0.36 & 0.39 \\
\hline 0.40 & 0.14 & 0.18 & 0.20 & 0.22 & 0.23 & 0.25 & 0.26 & 0.27 & 0.28 & 0.30 & 0.32 & 0.34 & 0.37 \\
\hline 0.30 & 0.12 & 0.15 & 0.17 & 0.20 & 0.21 & 0.22 & 0.24 & 0.25 & 0.26 & 0.28 & 0.30 & 0.32 & 0.35 \\
\hline 0.20 & 0.10 & 0.13 & 0.15 & 0.17 & 0.19 & 0.20 & 0.21 & 0.23 & 0.24 & 0.26 & 0.28 & 0.30 & 0.34 \\
\hline 0.10 & 0.08 & 0.11 & 0.13 & 0.15 & 0.16 & 0.18 & 0.19 & 0.20 & 0.22 & 0.24 & 0.26 & 0.28 & 0.32 \\
\hline 0.00 & 0.05 & 0.09 & 0.10 & 0.13 & 0.14 & 0.16 & 0.17 & 0.18 & 0.20 & 0.21 & 0.24 & 0.26 & 0.30 \\
\hline
\end{tabular}

Table 4: Calculations of the SPG for the ESF and ALBC examples for $D_{0}=0.25$

If Table 4 values are represented by rows, the first SPG is obtained (see Figure 3); and if Table 4 values are represented by columns, a second SPG is also obtained (see Figure 4). 


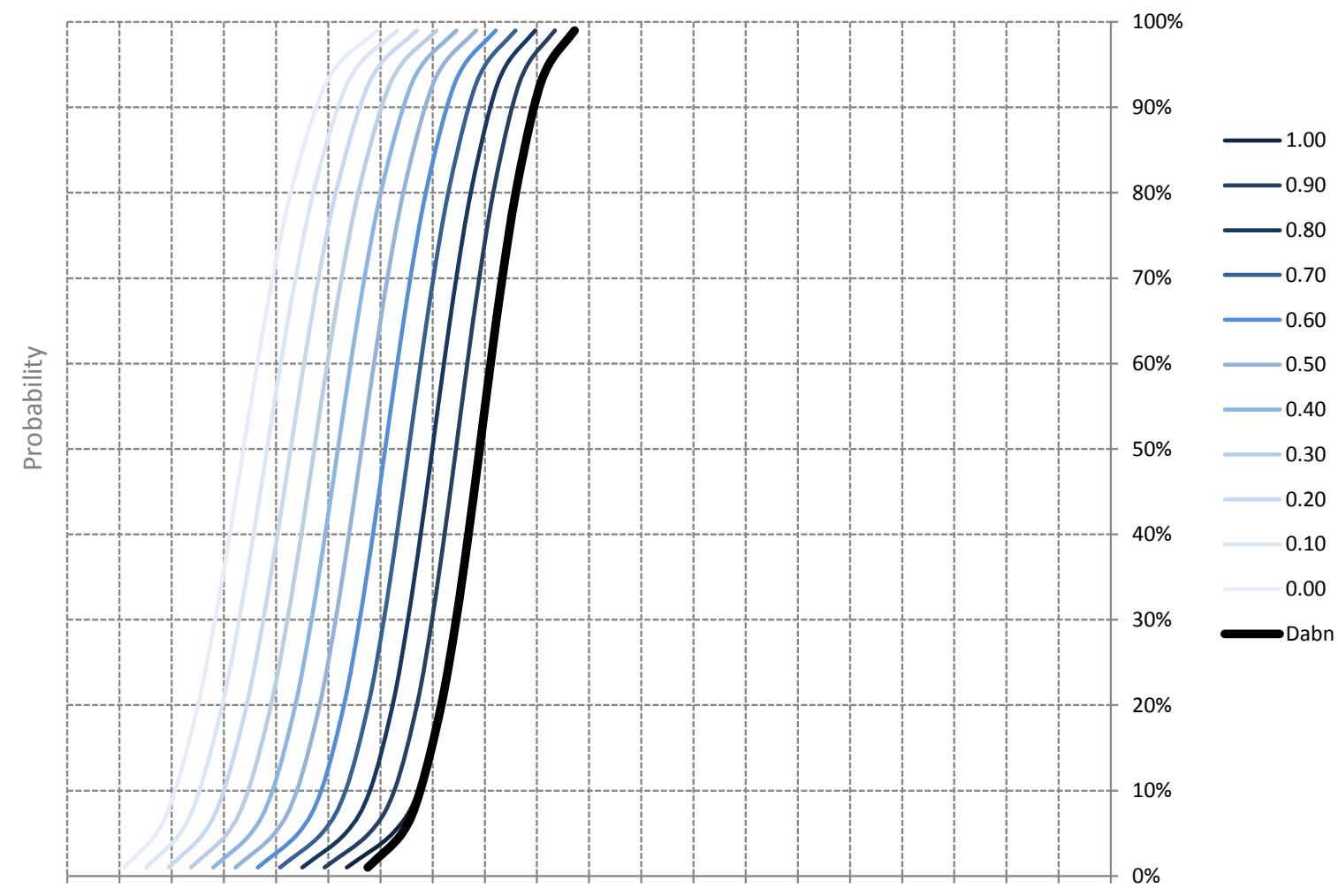

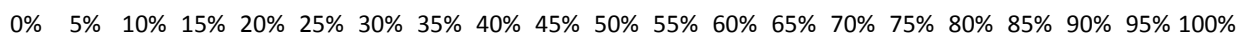

$\mathrm{Di}$

Figure 3: SPG in which curves represent different possible scores

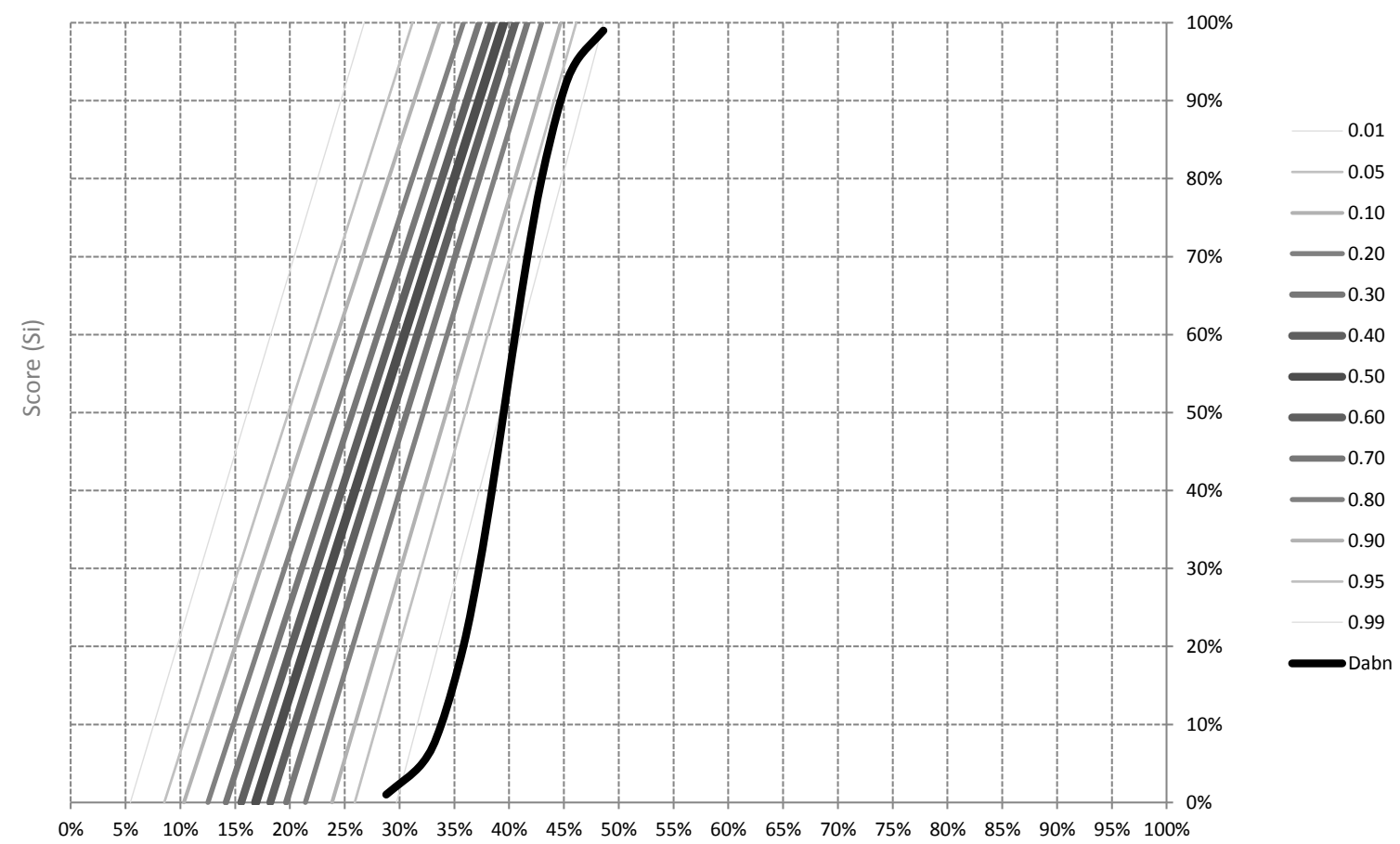

Figure 4: SPG in which curves represent various probable scoring distribution curves 
The graph in Figure 3 enables any bidder to discover, for every possible Drop (price discount, on axis $\mathrm{X}$ ), the probabilities it has of surpassing any possible score and of surpassing the abnormally high drop threshold. This graph constitutes the core of the BTFM, since its simplicity enables easy reading while including valuable processed tendering data.

The graph in Figure 4 represents the same variable on axis $X$, i.e., $D_{\text {i., }}$ but this time the $Y$ axis represents scores, which means that every curve shown (except for the $D_{a b n}$ curve which coincides with the previous SPG) represents the various probable scoring distribution curves that the final group of bids is likely to produce (the thicker a curve the more likely the real scoring distribution curve will occupy that position once the bids are opened and known).

\section{Drawing the Position Probability Graph (PPG)}

PPG constitutes a further step once the previous graphs have been calculated.

\subsection{Estimation of number of potential bidders}

To determine the probable positions each competitor will occupy it is necessary to delimit the number of potential bidders that will probably bid in the future tender. Indeed, an extensive literature has focused on the study of the potential number of bidders (Carr, 2005; Banki et al, 2008; Ngai et al., 2002); however, no variable has yet been proven to be reliable enough to forecast the number of bidders that will take part in a future tender.

Therefore, the BTFM will consider the variable 'number of bidders' $(\mathrm{N})$ as a random variable. Runeson, 1988, proved that when homogeneous tenders are analyzed, $\mathrm{N}$ follows a Normal distribution and can be delimited through average and standard deviation values of Ns from previous tenders (see last column in Table 1).

The necessary next step is to study the bids distances from each other, so that the various competitor positions can be forecasted. Concerning this particular problem, the BTFM has two advantages that previous models do not have: firstly, the limits of the maximum $\left(D_{\max }\right)$ and minimum $\left(D_{\min }\right)$ drops can be statistically determined; and, secondly, it is known the drop value $\left(D_{m}\right)$ that will cut in half the bids distribution.

Moreover, a simplifying assumption must be made: inside each range, the N/2 bidders will be placed equidistantly at the same probability intervals. Figure 5 shows that the relationship between bidder position $(n)$ and probability of surpassing the bidders' drops $\left(P_{n t h}\right)$ while Figure 6 shows the relationship between the bidders' drops $\left(D_{\text {nth }}\right)$ and the aforementioned probability $P_{n t h}$. 


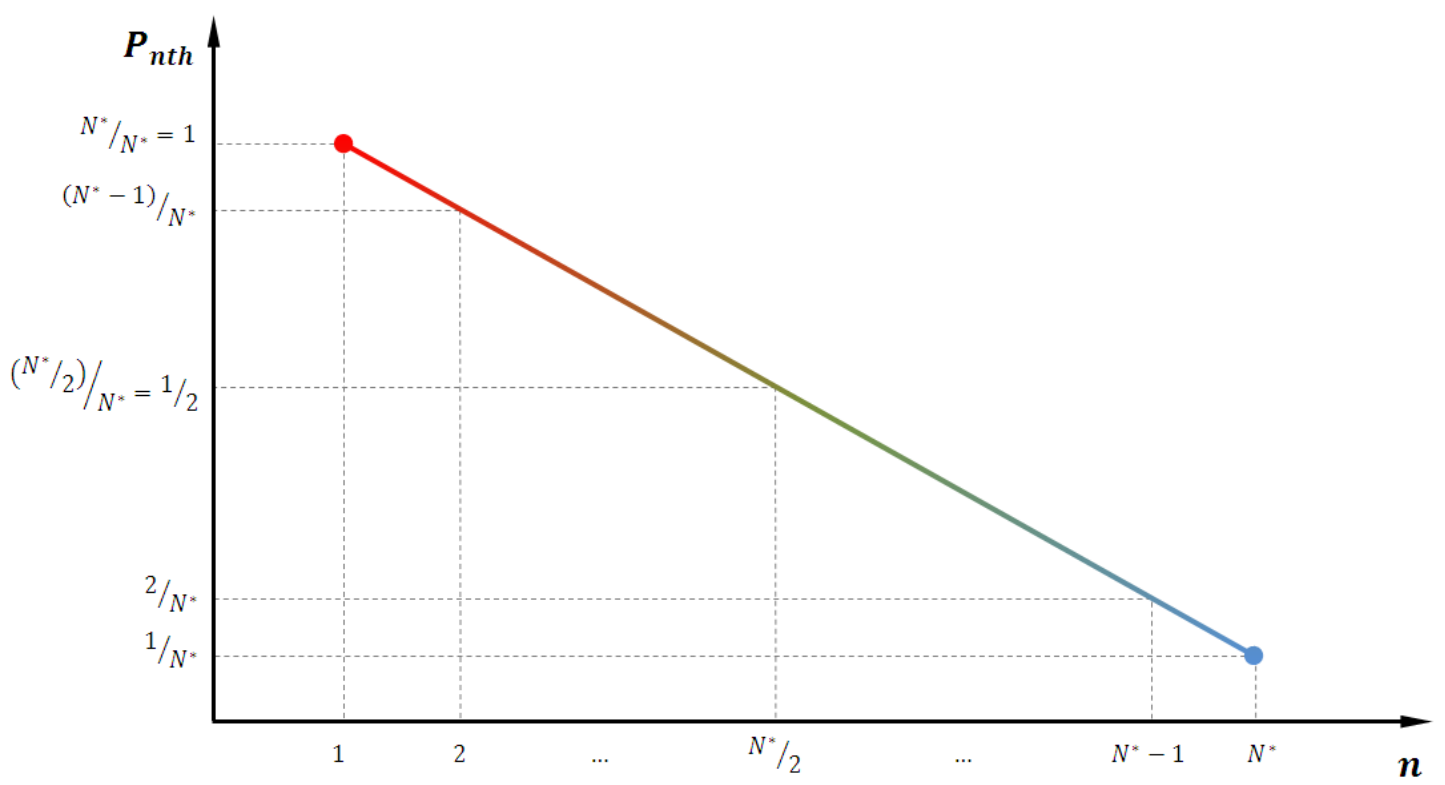

Figure 5: Diagram showing the relationship between $n$ and $P_{n t h}$.

The straight line represented in Figure 5 corresponds to this expression:

$$
P_{n t h}=\frac{N^{*}-n+1}{N^{*}}
$$

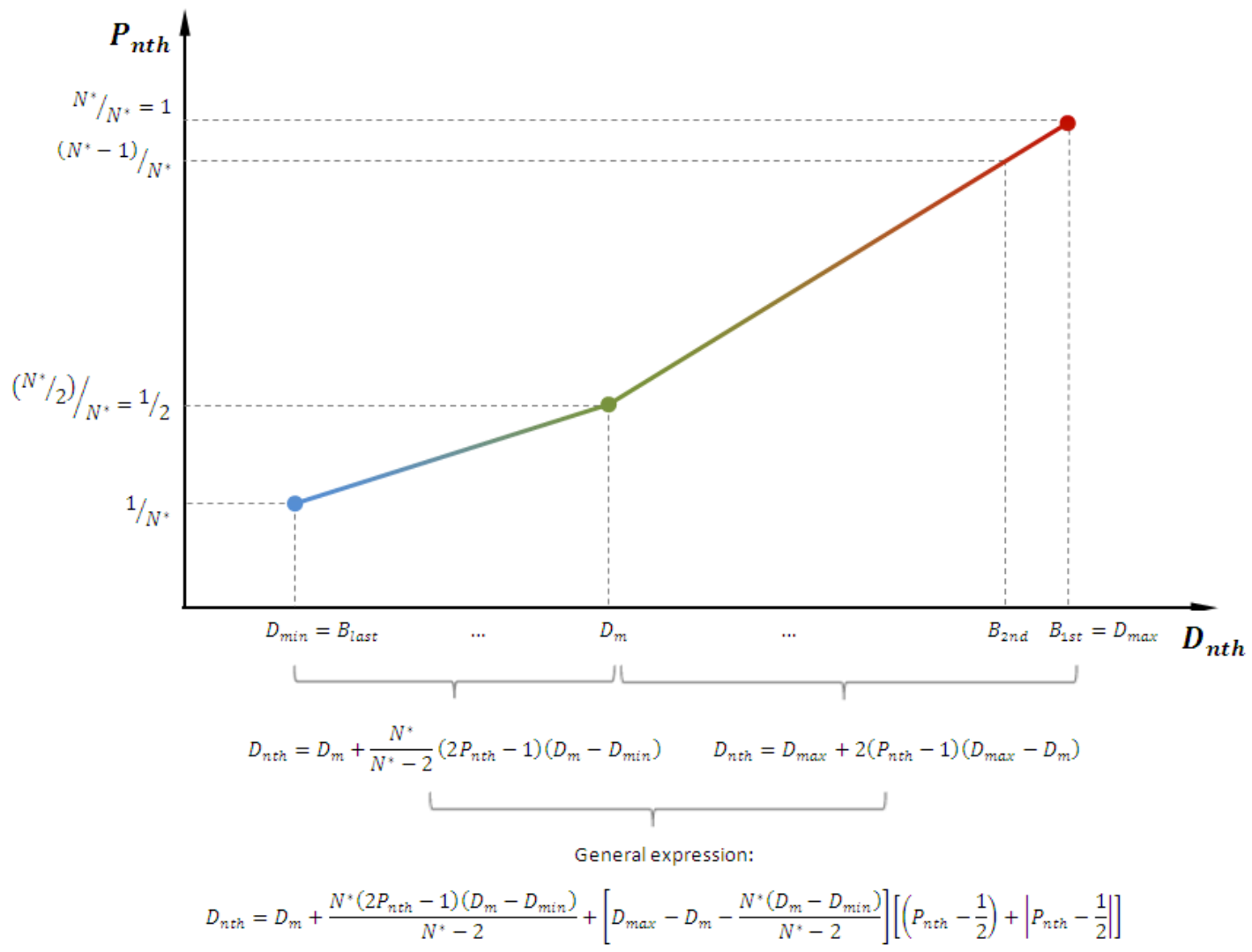

Figure 6: Diagram showing the relationship between $D_{\text {nth }}$ and $P_{\text {nth. }}$. 
The apparently simple linear and bi-linear distribution of bidders' drops in Figures 5 and 6, respectively, has also been studied in Ballesteros-Pérez, 2010, and the correlation factors $(R)$ obtained between real Bidders' drops and estimated bidders equidistant drops are almost always above 0.95 , meaning that the bi-linear bidders' distribution model is simple and accurate.

\subsection{Calculating and representing bidders position curves}

Once variables $D_{\min }, D_{m}, D_{\max }$ and $N^{*}$ are known for each possible probability level, it is assumed that the potential number of bidders will follow a bi-linear distribution as represented in figure 6 . The task remains of calculating each bidder's $n$ position curve by applying the general expressions shown below Figure 6, or its simplified equation (combination of equations 7 and the equation shown below Figure 6) as follows:

$$
\begin{gathered}
D_{n t h}=D_{m}+\frac{N^{*}-2 n+2}{N^{*}-2}\left(D_{m}-D_{\text {min }}\right)+ \\
+\left[\frac{D_{\max }-D_{m}}{2 N^{*}}-\frac{D_{m}-D_{\min }}{2\left(N^{*}-2\right)}\right]\left[\left(N^{*}-2 n+2\right)+\left|N^{*}-2 n+2\right|\right]
\end{gathered}
$$

Calculations using equation 8 for the first 25 potential bidders' positions are shown in Table 5 and represented in Figure 7. The only precaution that must be taken is not to use $\mathrm{N}^{*}$ values lower than 2 in order to maintain equation consistency.

This last type of graph enables any bidder to study which positions are the most attractive as a function of any possible drop (bid), since, quite often, the first positions involve high risks of disqualification because of the ALBC (as happens to the first and second bidders represented in figure 7 because they have most of their curves to the right of $D_{a b n}$ ).

In the example analyzed, a third, fourth or even fifth position would be more attractive since they would become first, second, and third positions, respectively, once the riskier bidders were eliminated. Therefore, this graph gives complementary information regarding the data given by the Scoring Probability Graphs. 


\begin{tabular}{|c|c|c|c|c|c|c|c|c|c|c|c|c|c|}
\hline$\downarrow S P$ Prob $\rightarrow$ & 0,01 & 0,05 & 0,10 & 0,20 & 0,30 & 0,40 & 0,50 & 0,60 & 0,70 & 0,80 & 0,90 & 0,95 & 0,99 \\
\hline Dmax & 0,27 & 0,31 & 0,34 & 0,37 & 0,39 & 0,41 & 0,42 & 0,44 & 0,46 & 0,48 & 0,52 & 0,54 & 0,59 \\
\hline $\mathrm{Dm}$ & 0,22 & 0,25 & 0,26 & 0,27 & 0,28 & 0,29 & 0,30 & 0,31 & 0,32 & 0,32 & 0,34 & 0,35 & 0,36 \\
\hline Dmin & 0,05 & 0,09 & 0,10 & 0,13 & 0,14 & 0,16 & 0,17 & 0,18 & 0,20 & 0,21 & 0,24 & 0,26 & 0,30 \\
\hline Dabn & 0,29 & 0,32 & 0,34 & 0,36 & 0,37 & 0,38 & 0,40 & 0,41 & 0,42 & 0,43 & 0,45 & 0,46 & 0,49 \\
\hline
\end{tabular}

\begin{tabular}{|c|c|c|c|c|c|c|c|c|c|c|c|c|c|}
\hline \multicolumn{14}{|c|}{ Total number of Bidders' Estimate $\left(\mathrm{N}^{*}\right)$} \\
\hline $\mathrm{Nm}$ & 13,89 & 13,89 & 13,89 & 13,89 & 13,89 & 13,89 & 13,89 & 13,89 & 13,89 & 13,89 & 13,89 & 13,89 & 13,89 \\
\hline h (1 variable total) & 1,00 & 1,00 & 1,00 & 1,00 & 1,00 & 1,00 & 1,00 & 1,00 & 1,00 & 1,00 & 1,00 & 1,00 & 1,00 \\
\hline$n_{0}$ & $-2,05$ & $-1,65$ & $-1,28$ & $-0,84$ & $-0,52$ & $-0,25$ & 0,00 & 0,25 & 0,52 & 0,84 & 1,28 & 1,65 & 2,32 \\
\hline$N_{\sigma}$ & 5,28 & 5,28 & 5,28 & 5,28 & 5,28 & 5,28 & 5,28 & 5,28 & 5,28 & 5,28 & 5,28 & 5,28 & 5,28 \\
\hline$h \cdot n_{\sigma} \cdot N_{\sigma}$ & 3,07 & 5,18 & 7,13 & 9,46 & 11,14 & 12,57 & 13,89 & 15,21 & 16,63 & 18,32 & 20,65 & 22,60 & 26,13 \\
\hline
\end{tabular}

\begin{tabular}{|c|c|c|c|c|c|c|c|c|c|c|c|c|c|c|}
\hline & & \multicolumn{13}{|c|}{ Dnth } \\
\hline$\downarrow$ Bidders & $\downarrow$ n Bidder Prob $\rightarrow$ & 0,02 & 0,05 & 0,10 & 0,20 & 0,30 & 0,40 & 0,50 & 0,60 & 0,70 & 0,80 & 0,90 & 0,95 & 0,99 \\
\hline $1 \mathrm{st}=\mathrm{Dmax}$ & 1 & 0,27 & 0,31 & 0,34 & 0,37 & 0,39 & 0,41 & 0,42 & 0,44 & 0,46 & 0,48 & 0,52 & 0,54 & 0,59 \\
\hline 2nd & 2 & 0,24 & 0,29 & 0,32 & 0,35 & 0,37 & 0,39 & 0,41 & 0,42 & 0,44 & 0,47 & 0,50 & 0,52 & 0,57 \\
\hline $3 r d$ & 3 & 0,08 & 0,26 & 0,29 & 0,33 & 0,35 & 0,37 & 0,39 & 0,41 & 0,43 & 0,45 & 0,48 & 0,51 & 0,56 \\
\hline 4th & 4 & $-0,24$ & 0,21 & 0,27 & 0,31 & 0,33 & 0,35 & 0,37 & 0,39 & 0,41 & 0,43 & 0,46 & 0,49 & 0,54 \\
\hline 5th & 5 & $-0,56$ & 0,10 & 0,23 & 0,29 & 0,31 & 0,33 & 0,35 & 0,37 & 0,39 & 0,41 & 0,45 & 0,47 & 0,52 \\
\hline 6th & 6 & $-0,88$ & 0,00 & 0,17 & 0,26 & 0,30 & 0,32 & 0,34 & 0,35 & 0,37 & 0,40 & 0,43 & 0,46 & 0,50 \\
\hline 7th & 7 & $-1,20$ & $-0,10$ & 0,11 & 0,22 & 0,27 & 0,30 & 0,32 & 0,34 & 0,36 & 0,38 & 0,41 & 0,44 & 0,49 \\
\hline 8th & 8 & $-1,52$ & $-0,20$ & 0,05 & 0,18 & 0,24 & 0,27 & 0,30 & 0,32 & 0,34 & 0,36 & 0,39 & 0,42 & 0,47 \\
\hline 9th & 9 & $-1,84$ & $-0,31$ & $-0,01$ & 0,14 & 0,21 & 0,25 & 0,28 & 0,30 & 0,32 & 0,34 & 0,38 & 0,40 & 0,45 \\
\hline 10th & 10 & $-2,16$ & $-0,41$ & $-0,07$ & 0,10 & 0,18 & 0,22 & 0,25 & 0,28 & 0,30 & 0,33 & 0,36 & 0,39 & 0,43 \\
\hline 11th & 11 & $-2,48$ & $-0,51$ & $-0,13$ & 0,06 & 0,15 & 0,20 & 0,23 & 0,26 & 0,29 & 0,31 & 0,34 & 0,37 & 0,42 \\
\hline 12th & 12 & $-2,80$ & $-0,61$ & $-0,20$ & 0,02 & 0,11 & 0,17 & 0,21 & 0,24 & 0,27 & 0,30 & 0,33 & 0,35 & 0,40 \\
\hline 13th & 13 & $-3,12$ & $-0,72$ & $-0,26$ & $-0,02$ & 0,08 & 0,14 & 0,19 & 0,22 & 0,26 & 0,29 & 0,32 & 0,34 & 0,38 \\
\hline 14th & 14 & $-3,44$ & $-0,82$ & $-0,32$ & $-0,06$ & 0,05 & 0,12 & 0,17 & 0,21 & 0,24 & 0,27 & 0,31 & 0,33 & 0,36 \\
\hline 15th & 15 & $-3,76$ & $-0,92$ & $-0,38$ & $-0,10$ & 0,02 & 0,09 & 0,14 & 0,19 & 0,22 & 0,26 & 0,30 & 0,32 & 0,36 \\
\hline 16th & 16 & $-4,08$ & $-1,02$ & $-0,44$ & $-0,14$ & $-0,01$ & 0,07 & 0,12 & 0,17 & 0,21 & 0,25 & 0,29 & 0,31 & 0,35 \\
\hline 17 th & 17 & $-4,40$ & $-1,13$ & $-0,50$ & $-0,18$ & $-0,04$ & 0,04 & 0,10 & 0,15 & 0,19 & 0,23 & 0,28 & 0,31 & 0,35 \\
\hline 18th & 18 & $-4,71$ & $-1,23$ & $-0,56$ & $-0,22$ & $-0,07$ & 0,01 & 0,08 & 0,13 & 0,17 & 0,22 & 0,27 & 0,30 & 0,34 \\
\hline 19th & 19 & $-5,03$ & $-1,33$ & $-0,63$ & $-0,26$ & $-0,10$ & $-0,01$ & 0,06 & 0,11 & 0,16 & 0,20 & 0,26 & 0,29 & 0,34 \\
\hline 20th & 20 & $-5,35$ & $-1,43$ & $-0,69$ & $-0,30$ & $-0,14$ & $-0,04$ & 0,03 & 0,09 & 0,14 & 0,19 & 0,25 & 0,28 & 0,33 \\
\hline $21 \mathrm{st}$ & 21 & $-5,67$ & $-1,54$ & $-0,75$ & $-0,34$ & $-0,17$ & $-0,06$ & 0,01 & 0,07 & 0,13 & 0,18 & 0,23 & 0,27 & 0,33 \\
\hline 22nd & 22 & $-5,99$ & $-1,64$ & $-0,81$ & $-0,38$ & $-0,20$ & $-0,09$ & $-0,01$ & 0,05 & 0,11 & 0,16 & 0,22 & 0,26 & 0,32 \\
\hline $23 r d$ & 23 & $-6,31$ & $-1,74$ & $-0,87$ & $-0,42$ & $-0,23$ & $-0,12$ & $-0,03$ & 0,03 & 0,09 & 0,15 & 0,21 & 0,26 & 0,31 \\
\hline 24th & 24 & $-6,63$ & $-1,84$ & $-0,93$ & $-0,46$ & $-0,26$ & $-0,14$ & $-0,06$ & 0,01 & 0,08 & 0,14 & 0,20 & 0,25 & 0,31 \\
\hline 25th & 25 & $-6,95$ & $-1,95$ & $-0,99$ & $-0,50$ & $-0,29$ & $-0,17$ & $-0,08$ & 0,00 & 0,06 & 0,12 & 0,19 & 0,24 & 0,30 \\
\hline
\end{tabular}

Table 5: Calculations of the PPG for the ESF and ALBC examples for $D_{0}=0.25$

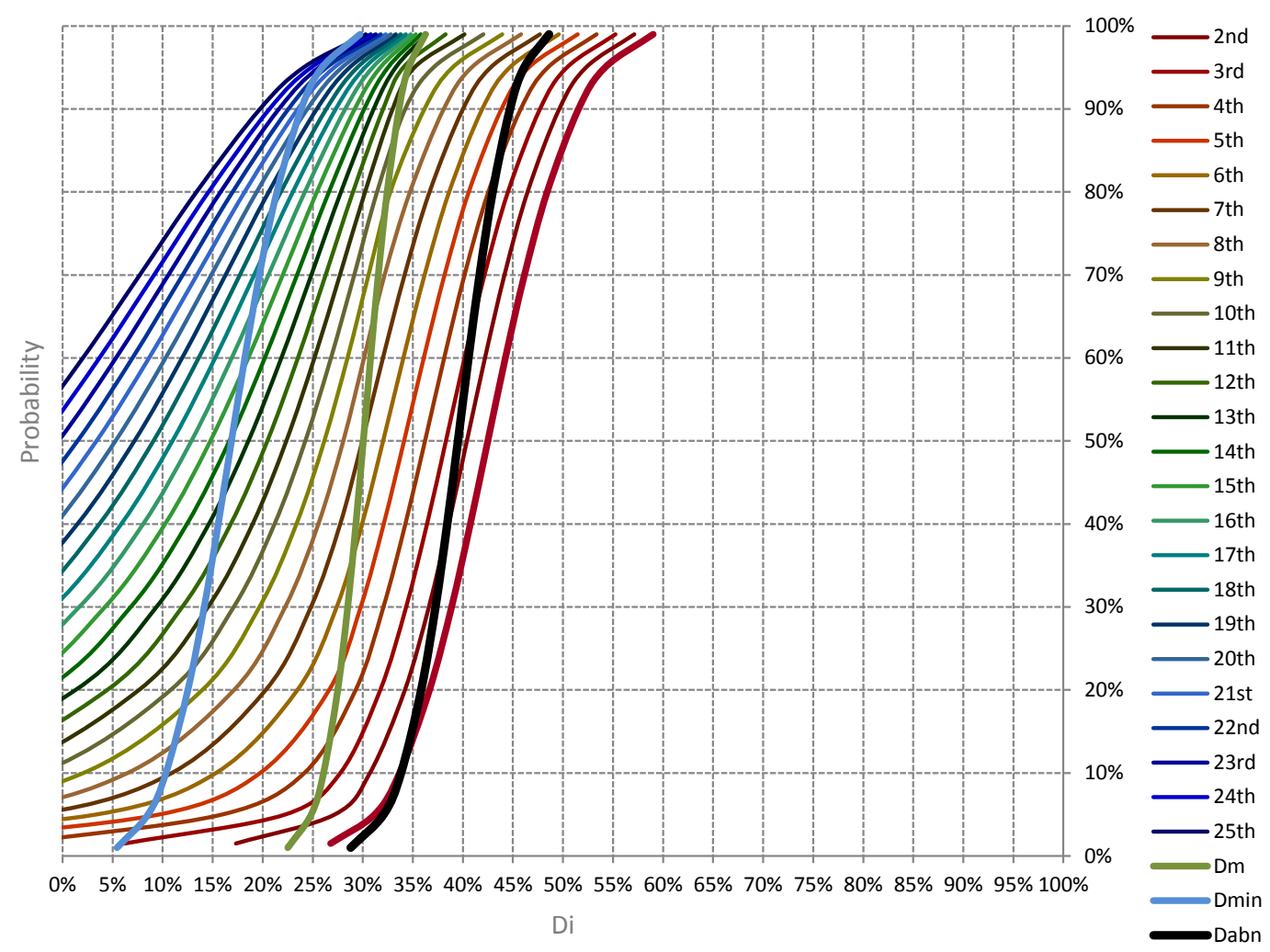

Figure 7: Position Probability Graph for the ESF and ALBC examples and for $D_{0}=0.25$ 


\section{Results}

In this paper, Ballesteros-Pérez et al., 2012a and 2012b's Bid tender forecasting model has been completed by generating two new kinds of graphs very useful for bidding manager when trying to make predictions. So far, none of the previous models have managed to calculate and briefly present in a graphical manner as much information as the presented model.

The whole BTFM is the sum of four graphs:

1. The iso-Score Curves Graph (iSCG) represents how a particular ESF distributes the economic points in a certain tender and represents the relationships between the main Scoring Parameters involved. There is no need for previous tender $D_{0}$ values, because it is a merely descriptive tool. Once the user is trained in its use, the iSCG highlights potential bidding strategies that the bidders will probably deploy to be more competitive.

2. The Scoring Probability Graphs (SPG), by means of two representations form the major outcome of the BTFM. These complementary and easy-to-use graphs enable any bidder to measure the probabilities of obtaining any economic score as a function of any bid. Moreover, they provide indispensable information regarding the likely limits for abnormally low bid thresholds.

3. The Position Probability Graph (PPG) enables the study of the problem from the perspective of the likely positions, that is, it takes into consideration the previous encounters with the competition concerning the number of bidders and its distribution. This graph enables bidders to identify the most desirable positions taking into account that a minimum economic score is not always enough and that it is usually necessary to occupy one of the first and 'accepted' positions to be sufficiently competitive.

\section{Conclusions and discussion}

Previous models were based on probabilistic descriptions of large groups of single bidders where each potential bidder was studied individually (it was even necessary to know the bidders' names and have an enormous amount of previous information with regard to their bids). The extended BTFM describes group patterns as a whole (using a significantly smaller dataset) and the model discretizes group bidder behavior to study the most likely positions only when bidder positions must be studied.

Nevertheless, there is a drawback: no analysis can be developed regarding how a specific bidder (a particular company for instance) will bid, however, the Ballesteros-Pérez et al. 
2012a and 2012b's BTFM, whose new graphs have been shown here, solves the major problems suffered by previous models in capped tendering as it enables us to:

1. Study bidding behaviors with a significantly smaller database compared to previous works, in which it was necessary to gather tens of previous tenders per each potential competitor bidder to be able to process useful statistical behaviors. In the proposed BTFM, the iSCG needs no previous tender experience, and the rest of the graphs can be fully operative with at least three previous tenders whenever they are homogeneous with the tender to be forecasted (similar type of work, ESF, location, and relatively similar budget). In other words it is no necessary to have shared a tender process with a particular competitor bidder, nor to try to predict which specific bidders will bid to be forecasted.

2. Forecast the probability of obtaining a particular score and/or position among competitors. The previous models were only able to study the likelihood of occupying the first position and did not study likely economic scorings, nor the likely thresholds of abnormally low bids.

3. Analyze time variations between tenders. Although this has not been explained in this paper, it is easy to reach the conclusion that time variations are easily studied by means of regression coefficient $(a, b, c$ and $d)$ variations. If these coefficient values are represented on a $\mathrm{Y}$-axis while $\mathrm{X}$-axis represents deadline dates of every closed tender, their trends and evolution (as multiples of each respective standard deviation coefficient, for instance) can be easily identified and quantified.

4. Measure the performance of a tender forecast. Previous models did not allow knowing in detail how close the tender outcome was to the initial prediction, and only Wallace, Patrick and Dowe (Skitmore, 2002) on their articles about 'mathematics and computers in sports' started to develop a 'Scoring logit function' combined with a new form of binomial test to tackle this particular issue of assessing forecast performance. The present model allows any tender manager to measure the accuracy of every parameter used in it. This can be easily done calculating how many standard deviation multiples of each coefficient $\left(a, b, c\right.$ and $d$ for assessing $D_{\max }, D_{\min }, \sigma$ and $D_{0}$, respectively) differ from their respective mean value (as calculated in Table 1) once the tender results are opened and the final bidders' positions and scores are known.

Finally, the BTFM completed by means of the SPG and the PPG is a very powerful tool for any Project or Tender Manager who wants to enter a bid and needs gaining an additional competitive edge. Nowadays, lots of companies compete to increase their project portfolios, 
and, the larger the number of companies that compete, the more difficult is to be awarded a contract.

The BTFM developed in this article allows a Project Manager to handle either vast or small amounts of previous tender data (calculations can be gradually developed using a simple spreadsheet, as demonstrated in the subsequent tables) and rapidly visualize the results represented in four graphs.

In a nutshell, those graphs enable the Project Manager to know, for every possible economic bid, which are the probabilities of occupying the first, second, and subsequent positions; the probabilities of achieving any particular score, and even the probabilities of being rejected because of an abnormal bid. Nonetheless, the final decision on which specific bid to propose (or even the final decision of not bidding) will depend on the Project Manager's risk conception, company profile and market conditions.

\section{Future work}

The BTFM set of equations implemented is not yet universal, as it has only been applied on capped tendering. In this kind of tenders, bidders can only underbid an initial monetary amount pre-set by the Contracting Authority, so every bid can be represented as a 'Drop' (or Discount) value. However, for non-capped tendering, as it is not possible to represent mathematical relationships in Drop values, the X-axis of the four graphs shown in this paper should represent monetary-based bids (ranging from 0 to infinite) instead of Drop values (in percentage or per-unit values, ranging from 0 to 1 , as used in this paper).

This change will also involve studying new mathematical relationships between Scoring and Forecasting Parameters the current model uses and transforming them as a function of monetary values instead of drop values, which is indispensable on non-capped tendering. This step will require access to non-capped tenders databases and further statistical analyses, nevertheless the adaptation of the model to cope with these new conditions can be researched.

In parallel with BTFM adaptation to non-capped tenders, a comprehensive study on how different Economic Scoring Formulas (the mathematical criteria by which scores are given out in a tender according to each bidder's bid, plus the way the abnormally low threshold is set as well) produce changes in the bidder behavior will also be made. With just a little experience in an ESF proposed for the first time by a contracting authority, the future BTFM will be able to make a relatively accurate forecast beforehand.

Finally, it was explained that the bid tender forecasting graphs shown here do not include certain risk issues and so a further step will be required to complement the variable 
deviations studied in this paper (particularly those related to the Estimated Cost, $\mathrm{D}_{0}$ ). For instance, there is an extensive literature regarding $D_{0}$ determination, and its connection with the present model will be researched to improve accuracy.

\section{Appendix A}

Main abbreviations used in the text:

A Amount of money of a tender (upper price limit)

a Regression coefficient to adjust relationship between $D_{\max }$ and $D_{m}$

$a^{*} \quad$ Variable calculated according to the expression $a_{m}+h \cdot n_{\sigma} \cdot a_{\sigma}$

$a_{m} \quad$ Average of regression coefficient a values

$\mathrm{a}_{\sigma} \quad$ Standard deviation of regression coefficient a values

ACA Agencia Catalana del Agua (owner of the tendering sub-dataset analyzed)

ALB Abnormally Low Bid

ALBC Abnormally Low Bid Criteria

B Bid (expressed in monetary value)

b Regression coefficient to adjust relationship between $D_{\min }$ and $D_{m}$

$b^{*} \quad$ Variable calculated according to the expression $b_{m}+h \cdot n_{\sigma} \cdot b_{\sigma}$

$b_{m} \quad$ Average of regression coefficient $b$ values

$b_{\sigma} \quad$ Standard deviation of regression coefficient $b$ values

Babn Abnormally Low Bid Threshold (expressed in monetary value)

$B_{i} \quad$ Bidder's i Bid (expressed in monetary value)

$B_{m} \quad$ Mean Bid (expressed in monetary value)

$\mathrm{B}_{\max } \quad$ Highest Bid (expressed in monetary value)

$\mathrm{B}^{*}{ }_{\text {min }}$ Lowest but not Abnormally Low Bid (expressed in monetary value). It is equal to the maximum value between $B_{\min }$ and $B_{a b n}$.

$B_{\min } \quad$ Lowest Bid (expressed in monetary value)

$\mathrm{B}_{0} \quad$ Amount equivalent to the Estimated Cost (expressed in monetary value)

BTFM Bid Tender Forecasting Model

C Regression coefficient to adjust relationship between $\sigma$ and $D_{m}$ 
$c^{*} \quad$ Variable calculated according to the expression $c_{m}+h \cdot n_{\sigma} \cdot c_{\sigma}$

$\mathrm{C}_{\mathrm{m}} \quad$ Average of regression coefficient $\mathrm{c}$ values

$\mathrm{C}_{\sigma} \quad$ Standard deviation of regression coefficient $\mathrm{c}$ values

d Regression coefficient to adjust relationship between $D_{0}$ and $D_{m}$

$d^{*} \quad$ Variable calculated according to the expression $d_{m}+h \cdot n_{\sigma} \cdot d_{\sigma}$

$d_{m} \quad$ Average of regression coefficient $d$ values

$d_{\sigma} \quad$ Standard deviation of regression coefficient $d$ values

D Drop (expressed in per-unit value)

$D_{\text {abn }} \quad$ Abnormal Drop (expressed in per-unit value)

$D_{\mathrm{i}} \quad$ Bidder's $i$ Drop (expressed in per-unit value)

$D_{m} \quad$ Mean Drop (expressed in per-unit value)

$D_{\max } \quad$ Maximum Drop (expressed in per-unit value)

$D^{*}{ }_{\max }$ Highest but not Abnormally High Drop (expressed in per-unit value). It is equal to the minimum value between $D_{\max }$ and $D_{a b n}$.

$D_{\min } \quad$ Minimum Drop (expressed in per-unit value)

$D_{\text {nth }} \quad$ Bidder's nth Drop (expressed in per-unit value)

$\mathrm{D}_{0} \quad$ Drop equivalent to the Estimated Cost (expressed in per-unit value)

ESF Economic Scoring Formula

h Multivariate Normal Distribution Coefficient. If there is one variable $h=1$, and if there are two variables $(h=\sqrt{ } 2 / 2 \approx 0,71)$.

iSC iso-Score Curve

iSCG iso-Score Curves Graph

$\mathrm{N} \quad$ Total number of bidders for a particular tender

$N^{*} \quad$ Variable calculated according to the expression $N_{m}+h \cdot n_{\sigma} \cdot N_{\sigma}$

$\mathrm{N}_{\mathrm{m}} \quad$ Average of analyzed $\mathrm{N}$ values

$\mathrm{N}_{\sigma} \quad$ Standard deviation of analyzed $\mathrm{N}$ values

$\mathrm{n}_{\sigma} \quad$ Number of Standard Deviation multiples that are related to a particular accumulated Standard Normal Distribution probability

P Probability (usually expressed in per-unit value) 
$P_{\text {nth }} \quad$ Probability of surpassing bidder's nth (usually expressed in per-unit value)

S Bids Standard Deviation (expressed in monetary value)

$\mathrm{S}_{\mathrm{i}} \quad$ Score of Bidder $i$ (expressed either in points or in per-unit value)

SP Scoring Parameter

SPG Scoring Probability Graph

PPG Position Probability Graph

T Abnormally High Drop Threshold

WWTPWaste Water Treatment Plant

$\sigma \quad$ Drops Standard Deviation (expressed in per-unit value)

\section{Appendix B}

Main equations linking the different variables used in the text:

$$
\text { Bidder's i Drop } \quad D_{i}=1-\frac{B_{i}}{A}
$$

Relationship between SP monetary-based and Drop-based values:

$$
\begin{gathered}
B_{m}=\left(1-D_{m}\right) A \quad ; \quad B_{\min }=\left(1-D_{\max }\right) A \quad ; \quad B_{\max }=\left(1-D_{\min }\right) A \\
S=\sigma \cdot A ; \quad B_{a b n}=\left(1-D_{a b n}\right) A
\end{gathered}
$$

Regression equations between SPs and FPs:

$$
\begin{gathered}
D_{\max }=a D_{m}^{2}+(1-a) D_{m} \quad ; \quad D_{\min }=b D_{m}^{2}+(1-b) D_{m} \\
\sigma=c\left(D_{m}^{\frac{1}{3}}-D_{m}\right) \quad ; \quad D_{0}=1+d\left(D_{m}-1\right)
\end{gathered}
$$

Expressions for calculating regression equation coefficient values:

$$
a_{k}=\frac{D_{\max k}-D_{m k}}{D_{m k}{ }^{2}-D_{m k}} ; b_{k}=\frac{D_{\min k}-D_{m k}}{D_{m k}{ }^{2}-D_{m k}} ; c_{k}=\frac{\sigma_{k}}{D_{m}^{1 / 3}-D_{m}} ; d_{k}=\frac{D_{0 k}-1}{D_{m k}-1}
$$

BTFM regression expressions as a function of $D_{0}$ :

$$
\begin{gathered}
D_{m}=1+\frac{D_{o}-1}{d^{*}} \\
D_{\text {max }}=a^{*} D_{m}{ }^{2}+\left(1-a^{*}\right) D_{m}=a^{*}\left(1+\frac{D_{o}-1}{d^{*}}\right)^{2}+\left(1-a^{*}\right)\left(1+\frac{D_{o}-1}{d^{*}}\right) \\
D_{\text {min }}=b^{*} D_{m}{ }^{2}+\left(1-b^{*}\right) D_{m}=b^{*}\left(1+\frac{D_{o}-1}{d^{*}}\right)^{2}+\left(1-b^{*}\right)\left(1+\frac{D_{o}-1}{d^{*}}\right)
\end{gathered}
$$




$$
\sigma=c^{*}\left(D_{m}{ }^{1 / 3}-D_{m}\right)=c^{*}\left[\left(1+\frac{D_{o}-1}{d^{*}}\right)^{\frac{1}{3}}-\left(1+\frac{D_{o}-1}{d^{*}}\right)\right]
$$

Expressions for calculating asterisk regression coefficient values:

$$
\begin{array}{lll}
a^{*}=a_{m}+h \cdot n_{\sigma} \cdot a_{\sigma} \quad ; & b^{*}=b_{m}+h \cdot n_{\sigma} \cdot b_{\sigma} \\
c^{*}=c_{m}+h \cdot n_{\sigma} \cdot c_{\sigma} \quad ; & d^{*}=d_{m}+h \cdot n_{\sigma} \cdot d_{\sigma}
\end{array}
$$

ESF used in the example:

$$
\begin{gathered}
S_{i}=1-\frac{B_{i}-B_{\min }^{*}}{B_{\max }-B_{\min }^{*}} \rightarrow \quad S_{i}=1-\frac{D_{\text {max }}^{*}-D_{i}}{D_{\text {max }}^{*}-D_{\min }} \rightarrow \\
\rightarrow \quad D_{i}=D_{\text {max }}^{*}-\left(1-S_{i}\right)\left(D_{\text {max }}^{*}-D_{\text {min }}\right)
\end{gathered}
$$

ALBC used in the example:

$$
B_{a b n}=B_{m}-2 S \quad \rightarrow \quad D_{a b n}=D_{m}+2 \sigma
$$

Number of bidders for each probability level calculation:

$$
N^{*}=N_{m}+h \cdot n_{\sigma} \cdot N_{\sigma}
$$

BTFM relationship for probability $\left(P_{n \text {th }}\right)$, number of bidders $\left(N^{*}\right)$, and position $(n)$ :

$$
P_{n t h}=\frac{N^{*}-n+1}{N^{*}}
$$

BTFM relationship for nth's bidder Drop $\left(D_{n+t}\right)$, probability $\left(P_{n t h}\right)$, and number of bidders $\left(N^{*}\right)$ :

$$
\begin{aligned}
\text { For } \frac{1}{N^{*}} \leq P_{n t h} & \leq \frac{1}{2} \text { and } D_{\text {min }} \leq D_{n t h} \leq D_{m} \\
D_{n t h} & =D_{m}+\frac{N^{*}}{N^{*}-2}\left(2 P_{n t h}-1\right)\left(D_{m}-D_{m i n}\right)
\end{aligned}
$$$$
\text { For } \frac{1}{2} \leq P_{n t h} \leq 1 \text { and } D_{m} \leq D_{n t h} \leq D_{\max } \text { : }
$$

$$
D_{n t h}=D_{\max }+2\left(P_{n t h}-1\right)\left(D_{\max }-D_{m}\right)
$$

General expression (for $\frac{1}{N^{*}} \leq P_{n t h} \leq 1$ and $D_{\min } \leq D_{n t h} \leq D_{\max }$ ) 


$$
\begin{aligned}
D_{n t h}=D_{m} & +\frac{N^{*}\left(2 P_{n t h}-1\right)\left(D_{m}-D_{\min }\right)}{N^{*}-2} \\
& +\left[D_{\max }-D_{m}-\frac{N^{*}\left(D_{m}-D_{\min }\right)}{N^{*}-2}\right]\left[\left(P_{n t h}-\frac{1}{2}\right)+\left|P_{n t h}-\frac{1}{2}\right|\right]
\end{aligned}
$$

Or:

$$
\begin{aligned}
D_{n t h}=D_{m} & +\frac{N^{*}-2 n+2}{N^{*}-2}\left(D_{m}-D_{\text {min }}\right) \\
& +\left[\frac{D_{\max }-D_{m}}{2 N^{*}}-\frac{D_{m}-D_{\min }}{2\left(N^{*}-2\right)}\right]\left[\left(N^{*}-2 n+2\right)+\left|N^{*}-2 n+2\right|\right]
\end{aligned}
$$

\section{Acknowledgements}

On correcting this paper, the authors really appreciate the help provided by Professor Martin Skitmore from Queensland University of Technology.

The translation and revision of this paper was funded by the Universitat Politècnica de València.

\section{References}

Ballesteros-Pérez, P, 2010. Doctoral thesis: "Propuesta de un nuevo modelo para la predicción de bajas en licitaciones de Construcción”. González Cruz, MC. dir. ; Pastor Ferrando, JP. dir. . Universal Identifier: http://hdl.handle.net/10251/7025

Ballesteros-Pérez, P., González-Cruz, Ma . C., Pastor-Ferrando, J.P. and Fernández-Diego, M. 2012. The iso-Score Curve Graph. A new tool for competitive bidding, Automation in Construction. 22 (1), 481-490.

Ballesteros-Pérez, P., González-Cruz, Ma. C., Cañavate-Grimal, A. 2012. Mathematical relationships between Scoring Parameters in Capped Tendering. International Journal of Project Management. 30 (7), 850-862.

Banki, M. T., Esmaeeli, B., Ravanshadnia, M., 2008. The assessment of bidding strategy of Iranian construction firms. International Journal of Management Science and Engineering Management, 4 (2), 153-160. 
Carr, P.G., 2005. Investigation of Bid price competition measured through prebid project estimates, actual bid prices and Number of bidders. Journal of Construction Engineering and Management. 131 (11), 1165-1172.

Carr, R.I., 1982. General bidding model Journal of the Construction Division, Proceedings of the American Society of Civil Engineers. 108 (CO4), 639-50.

Chao, L. and Liou, C., 2007. Risk-minimizing approach to bid-cutting limit determination. Construction Management and Economics. 25 (8), 835-843.

Deltas, G. and Engelbrecht-Wiggans, R., 2005. Naive Bidding. Management Science. 51 (3), 328-338.

Dikmen, I., Talat, M. and Kemal, A., 2007. A case-based decision support tool for bid markup estimation of international construction projects Automation in Construction, 17 (1) 30-44.

Drew, D. and Skitmore, R.M., 1997. The effect of contract type and size on competitiveness in bidding. Construction Management and Economics. 15, 469-489.

Egemen, M., Mohamed, A.N., 2007. A framework for contractors to reach strategically correct bid/no bid and mark-up size decisions. Building and Environment, 42 (3), 1373-1385.

Engelbrecht-Wiggans, R., 1980. State of the Art-Auctions and Bidding Models: A Survey. Management Science. 26 (2), 119-142.

Engelbrecht-Wiggans, R., 1989. The Effect of Regret on Optimal Bidding in Auctions. Management Science. 35 (6), 685-692.

Fayek, A., 1998. Competitive bidding strategy model and software system for bid preparation. Journal of Construction Engineering and Management. 124, 1-10.

Friedman, L., 1956. A competitive bidding strategy Operations Research 1 (4), 104-12.

Gates, M., 1967. Bidding strategies and probabilities Journal of the Construction Division, Proceedings of the American Society of Civil Engineers. 93 (CO1), 75-107.

Han, S.H; Diekmann, J.E., Ock J.H., 2005. Contractor's Risk Attitudes in the Selection of International Construction Projects. ASCE Journal of Construction Engineering and Management, 131 (3), 283-292.

Harstad, R.M. and Saša Pekec, A., 2008. Relevance to Practice and Auction Theory: A Memorial Essay for Michael Rothkopf. Interfaces. 38 (5) 367-380. 
Hartono, B. and Yap, C.M., 2011. Understanding risky bidding: a prospect-contingent perspective Construction Management and Economics. 29 (6), 579-593.

Laryea, S. and Hughes, W., 2011. Risk and Price in the Bidding Process of Contractors Journal of Construction Engineering and Management. 137 (4), 248-259.

Lo, W.; Lin, C.L. and Yan, M.R., 2007. Contractor's Opportunistic Bidding Behavior and Equilibrium Price Level in the Construction Market. Journal of Construction Engineering and Management. 133 (6), 409-416.

McCaffer, R. and Pettitt, A.N., 1976. Distribution of bids for buildings and road contracts Operational Research Quarterly. 27 (4i), 835-43.

Mitchell, M.S., 1977. The probability of being the lowest bidder Applied Statistics 2 (2), 1914.

Mohamed, K.A., Khoury, S.S. and Hafez, S.M., 2011. Contractor's decision for bid profit reduction within opportunistic bidding behavior of claims recovery International Journal of Project Management. 29 (1), 93-107.

Naoum, S.G., 1994. Critical Analysis of Time and Cost of Management and Traditional Contracts. Journal of Construction Engineering and Management. 120 (4), 687-705.

Näykki, P., 1976. On Optimal Bidding Strategies. Management Science. 23 (2), 198-203.

Ngai, S.C., Derek, S., Drew, H.P. and Skitmore, R.M. 2002. A theoretical framework for determining the minimum number of bidders in construction bidding competitions. Construction Management and Economics. 20 (6), 473-482.

Oo, B., Drew, D.S. and Runeson, G., 2010. Competitor analysis in construction bidding Construction Management and Economics, 28 (12), 1321-1329.

Oo, B.L., Drew, D.S. and Lo, H.P., 2008. A comparison of contractors' decision to bid behaviour according to different market environments International Journal of Project Management. 26 (4), 439-447.

Oo, B.L., Drew, D.S. and Lo, H.P., 2008. Heterogeneous Approach to Modeling Contractors' Decision-to-Bid Strategies Journal of Construction Engineering and Management. 134 (10), 766-776 
Pim, J.C., 1974. Competitive tendering and bidding strategy National Builder. 55 (11), 541 545.

Ravanshadnia, M., Rajaie, H., Abbasian, H.R., 2010. Hybrid fuzzy MADM project-selection model for diversified construction companies. Canadian Journal of Civil Engineering 37 (8), 1082-1093.

Rothkopf, M.H., 1969. A Model of Rational Competitive Bidding. Management Science. 15 (7) 362-373.

Rothkopf, M.H. and Harstad, R.M., 1994. Modeling Competitive Bidding: A Critical Essay. Management Science. 40 (3), 364-384.

Runeson, G., 1988. An analysis of the accuracy of estimating and the distribution of tenders. Construction Management and Economics, 6, 357-370.

Runeson, K.G. and Skitmore, R.M., 1999. Tendering theory revisited Construction Management and Economics. 17 (3), 285-96.

Skitmore, R.M., 1991. The contract bidder homogeneity assumption: an empirical analysis. Construction Management and Economics. 9 (5), 403-429.

Skitmore, R.M., 2002. The probability of tendering the lowest bid in sealed auctions: an empirical analysis of construction data. Journal of the Operational Research Society. 53 (1), 47-56.

Skitmore, R.M., 2004. Predicting the probability of winning sealed bid auctions: the effects of outliers on bidding models. Construction Management and Economics. 22 (1), $101-109$.

Skitmore, R.M., 2008. First and second price independent values sealed bid procurement auctions: some scalar equilibrium results Construction Management and Economics. 26 (8), 787-803.

Skitmore, R.M., Drew, D.S. and Ngai, S., 2001. Bid-spread. Journal of Construction Engineering and Management. 127 (2), 149-153.

Skitmore, R.M. and Runeson, G., 2006. Bidding models: testing the stationarity assumption Construction Management and Economics. 24 (8), 791-803.

Skitmore, R.M., Pettitt, A.N. and McVinish, R., 2007. Gates' Bidding Model. Journal of Construction Engineering and Management. 133 (11), 855-863. 
Stark, R.M. and Rothkopf, M.H., 1979. Competitive bidding: A comprehensive bibliography. Journal of the Operational Research. 27 (2), 364-390.

Vergara, A.J., 1977. Probabilistic estimating and applications of portfolio theory in construction. Ph.D. thesis, Department of Civil Engineering, University of Illinois at UrbanaChampaign, Urbana, III.

Watt, D.J., Kayis, B. and Willey, K., 2009. Identifying key factors in the evaluation of tenders for projects and services. International Journal of Project Management. 27 (3), 250-260.

Ye, K.; Jiang, W. and Shen, L., 2008. Project competition intensity (PCI) in the construction market: a case study in China. Construction Management and Economics. 26 (5) 463-470. 